\title{
Validation strategies for the interpretation of microstructure imaging using diffusion MRI
}

Dyrby, Tim Bjørn; Innocenti, Giorgio M.; Bech, Martin; Lundell, Henrik

Published in:

Neurolmage

Link to article, DOI:

10.1016/j.neuroimage.2018.06.049

Publication date:

2018

Document Version

Peer reviewed version

Link back to DTU Orbit

Citation (APA):

Dyrby, T. B., Innocenti, G. M., Bech, M., \& Lundell, H. (2018). Validation strategies for the interpretation of microstructure imaging using diffusion MRI. Neurolmage, 182, 62-79.

https://doi.org/10.1016/j.neuroimage.2018.06.049

\section{General rights}

Copyright and moral rights for the publications made accessible in the public portal are retained by the authors and/or other copyright owners and it is a condition of accessing publications that users recognise and abide by the legal requirements associated with these rights.

- Users may download and print one copy of any publication from the public portal for the purpose of private study or research.

- You may not further distribute the material or use it for any profit-making activity or commercial gain

- You may freely distribute the URL identifying the publication in the public portal 


\title{
Validation strategies for the interpretation of microstructure imaging using diffusion MRI
}

\author{
Tim B. Dyrby a,b, , Giorgio M. Innocenti ${ }^{\mathrm{c}, \mathrm{d}}$, Martin Bech $^{\mathrm{e}}$, Henrik Lundell $^{\mathrm{a}}$ \\ ${ }^{\text {a }}$ Danish Research Centre for Magnetic Resonance, Center for Functional and Diagnostic Imaging and Research, Copenhagen University Hospital Hvidovre, Hvidovre, \\ Denmark \\ b Department of Applied Mathematics and Computer Science, Technical University of Denmark, Kongens Lyngby, Denmark \\ ${ }^{\mathrm{c}}$ Karolinska Institutet, Department of Neuroscience, Stockholm, Sweden \\ ${ }^{\mathrm{d}}$ Brain and Mind Institute, Swiss Federal Institute of Technology in Lausanne, Lausanne, Switzerland \\ ${ }^{\mathrm{e}}$ Medical Radiation Physics, Lund University, Lund, Sweden
}

\section{A B S T R A C T}

Extracting microanatomical information beyond the image resolution of MRI would provide valuable tools for diagnostics and neuroscientific research. A number of mathematical models already suggest microstructural interpretations of diffusion MRI (dMRI) data. Examples of such microstructural features could be cell bodies and neurites, e.g. the axon's diameter or their orientational distribution for global connectivity analysis using tractography, and have previously only been possible to access through conventional histology of post mortem tissue or invasive biopsies. The prospect of gaining the same knowledge non-invasively from the whole living human brain could push the frontiers for the diagnosis of neurological and psychiatric diseases. It could also provide a general understanding of the development and natural variability in the healthy brain across a population. However, due to a limited image resolution, most of the dMRI measures are indirect estimations and may depend on the whole chain from experimental parameter settings to model assumptions and implementation.

Here, we review current literature in this field and highlight the integrative work across anatomical length scales that is needed to validate and trust a new dMRI method. We encourage interdisciplinary collaborations and data sharing in regards to applying and developing new validation techniques to improve the specificity of future dMRI methods.

\section{Introduction}

Microstructure imaging using diffusion MRI (dMRI) provides a sensitive probe to tissue structure on a micrometre $(\mu \mathrm{m})$ length scale with a wide range of applications ranging from estimating cell sizes and orientations to reconstructing axonal pathways across different brain regions. With an image resolution on the order of millimetres, dMRI entangles information from millions of cells of different types in different configurations or even contributions from different tissue types. The very indirect relation between the structure of individual cells and the diffusion process averaged over the image voxel calls for independent validation techniques. The choice of a validation technique is often guided by our expectations based on theory, simulations, or empirical MRI experiments. However, the true microstructural anatomy is complex, and the pros and cons of the individual validation techniques must therefore be carefully considered. As multiple anatomical effects, ranging from molecular scales to overall organization of cells, could influence dMRI, the relevant validation experiments should cover a broad range of anatomical sizes. Consequently, validation is an iterative process that may get increasingly sophisticated over the years as the method continues to develop. This dynamic validation process is also a driving factor behind the methodological evolution of dMRI, as many examples in literature demonstrate. For instance, it is well known that local fibre reconstruction models for tractography must account for fibre crossings for better extraction of some brain connections using e.g. structure tensor imaging and tracers for validation (Leergaard et al., 2010; Behrens et al., 2007; Budde and Annese, 2013). Similar development is currently ongoing in the methodological evolution of non-invasive axon diameter estimation using dMRI or g-ratio imaging with a combination of different quantitative MRI modalities (see review (Campbell et al., 2017)). Examples demonstrate that today's microstructural imaging using dMRI can be pushed towards a $\mu$ m-resolution of single cells under ultra-high field conditions and with small tissue samples (Hansen et al., 2010; Flint et al., 2010; Hansen et al., 2011), as well as the image resolution on whole organ samples ex vivo, or preclinical and clinical in vivo imaging range from sub-millimetre for high resolution imaging to several millimetres in

\footnotetext{
* Corresponding author. Danish Research Centre for Magnetic Resonance (DRCMR), Section 714, Center for Functional and Diagnostic Imaging and Research, Copenhagen University Hospital Hvidovre, Kettegård Allé 30, 2650 Hvidovre, Denmark.

E-mail address: timd@drcmr.dk (T.B. Dyrby).
} 
more clinically feasible settings. Most often, interpretations of dMRI derived measures are therefore far from a direct visualization of a specific microstructure but rather an indirect interpretation. In the context of choosing a suitable validation strategy, it is important to find imaging techniques for validation with sufficient overlap in terms of interpretation and to understand the possible ambiguities that might be generated by anatomical features not visible with the selected validation technique. Fig. 1 is a schematic illustration of the relation between anatomically length scales and the capabilities of various imaging methods, which can be used to investigate features of the different length scales directly or indirectly dependent on their image resolution. As shown in Fig. 1 (x-axis), different imaging methods may capture the same anatomical features (Fig. 1, y-axis) but from different perspectives using different measuring techniques or at different image resolutions.

The focus of this review is to give insight into different invasive imaging technologies and animal models that can be used to validate microstructural interpretations based on dMRI data. We discuss pros and cons of the different validation strategies based on Fig. 1, as well as the challenges in defining a gold standard for a complex anatomical environment that is still not fully understood. Diffusion-based models and dMRI sequence design will be discussed as being a black box to be validated for a hypothesized interpretation. The reader interested in underlying methodological details will be guided to relevant literature.

\section{Sensitizing MRI to microstructure}

\section{MRI tissue contrasts - understanding and quantifying effects}

MRI tissue contrasts can be related to a number of physical effects ranging from the molecular level, such as relaxation, magnetization transfer and chemical exchange, to cellular geometry and mesoscopic organization modulating the diffusion weighted signal. Today, most clinically relevant contrasts relate to effects that are readily seen directly in anatomical MRI, i.e. with a qualitative analysis. Many of those contrast mechanisms can be well established and very valuable in clinical use, like the strong dMRI contrast in tissue affected by ischemic stroke, although it is not yet fully understood how pathology modulates diffusivity in terms of microstructure (Moseley et al., 1990). This does not undermine the value of dMRI as a biomarker, but understanding the underlying mechanisms could thus be one topic of interest for validation. At the other end of contrast magnitudes are subtle changes in healthy (e.g. brain maturation) or normal appearing tissue in diseases as dementia and multiple sclerosis. To study those changes and in order to compare changes across different brain regions or over a population of subjects, the data must be quantitative to allow for statistical analyses. Fig. 2 demonstrates in vivo multimodal MRI using a clinical 3T scanner and includes both conventional (e.g. T1w, T2w and FLAIR), as well as examples of derived quantitative structural MRI, (i.e. relaxation maps and quantitative magnetization transfer) and different dMRI-based metrics. Quantitative MRI is important because one can measure a property of the tissue which to a high degree is independent of the properties of the scanner and measurement technique. A quantitative map relate to either one or more physical entities itself, e.g. relaxation time constants, diffusion coefficients, or biophysical features, like cell and neurite density, type of cells, restriction sizes, and architecture (see examples of taxometry of biophysical diffusion models in Panagiotaki et al., 2012). The physical and biophysical approaches are complementary. However, the biophysical model approach is attractive as it offers a clear interpretation of the MRI data, but the specificity to a microstructural feature is not guaranteed per se by a parameter name. It is tempting to account for many possible effects and to build more complex models, but the ambiguities include the risk of over-fitted models and over-interpreted results (Jelescu et al., 2016). One is already careful in the naming of model parameters by using words like "apparent", "index" or "estimation", as used in Apparent Diffusion Coefficient (ADC) (Basser et al., 1994), Apparent Exchange Rate (AXR) (Lasič et al., 2011) or Axon Diameter Index (ADI) (Alexander et al., 2010).

\section{Translational MRI}

As a non-invasive technique, MRI can be translated from animal to human applications. Preclinical MRI enables the combination with a range of independent invasive validation methods on the same specimens. Furthermore, its hardware is powerful and allows larger parameter spaces to be investigated than possible using clinical scanners. Longer scan times than those acceptable in in vivo human can often also be used, which can boost the quality of the data further. In the following, we highlight the variety of animals models suitable for different research topics and quality issues in in vivo versus ex vivo imaging.

\section{Animal models}

When deciding on a validation strategy using invasive techniques, a suitable animal model must be selected that reflects the microstructural features of interest. For translation to human use, the anatomical resemblance of the animal model can be focused upon basic microstructural features, e.g. cells and neurites, or larger anatomical structures

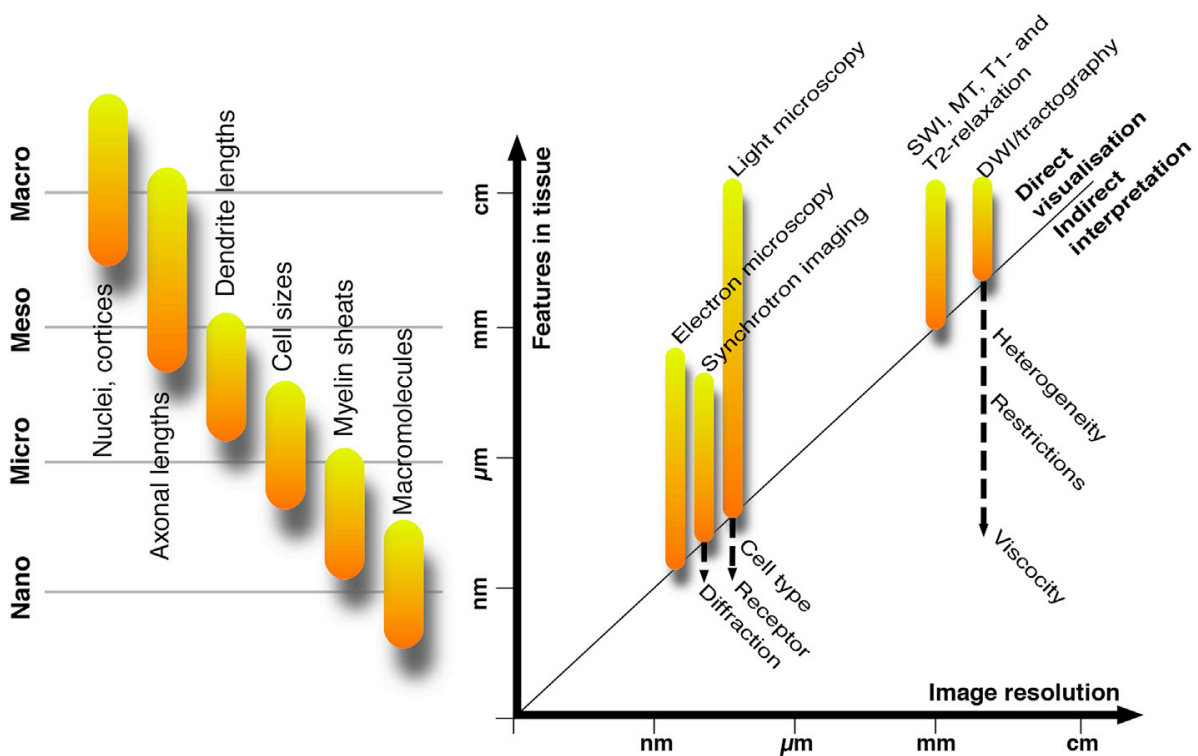

Fig. 1. Left panel shows a number of anatomical features characterized from nano-to macroscopic length scales. Right panel shows the relation between the image resolution (x-axis) and the size of features of interest in tissue (y-axis) for a number of imaging techniques. We distinguish between direct visualization of features visible at a given resolution (above the diagonal), and indirect interpretation of features reflected in the image contrast (below the diagonal). Non-invasive in vivo feasible MRI-based methods, dMRI, relaxation (T1 and T2), magnetization transfer (MT), and susceptibility weighted imaging (SWI)) have image resolution orders of magnitude lower than tissue invasive histological techniques (light and electron microscopy and synchrotron imaging). 

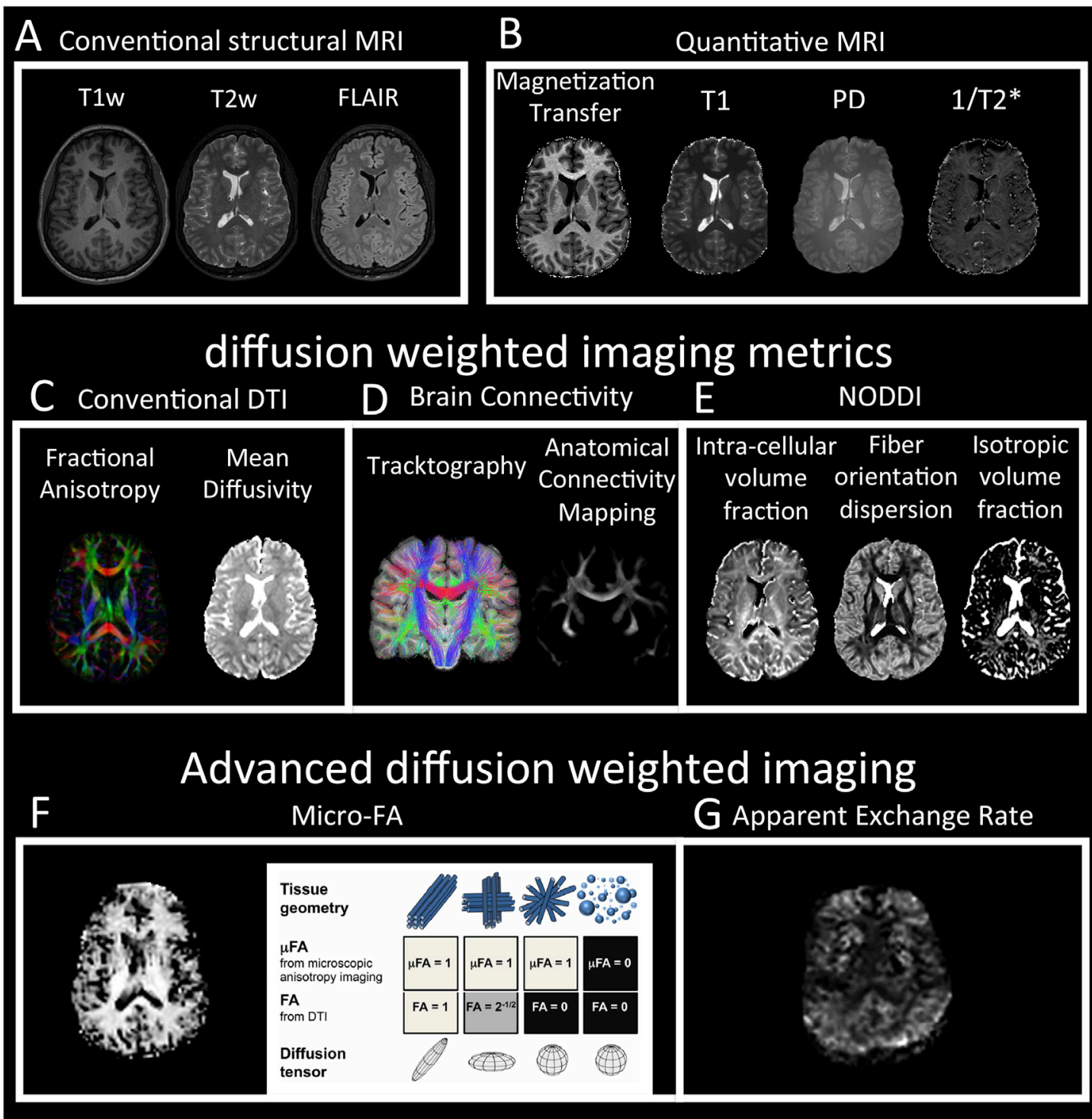

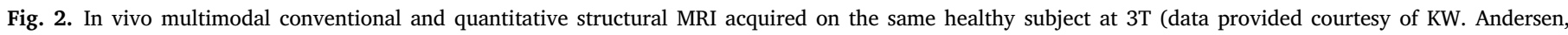

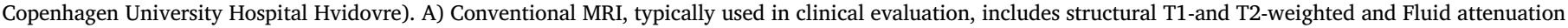

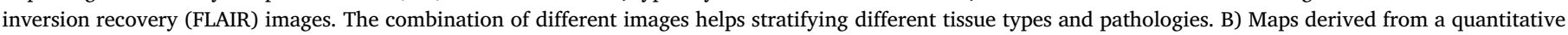

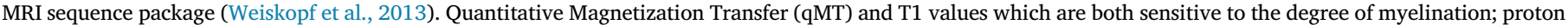

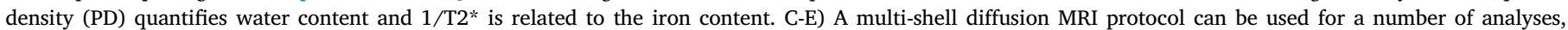

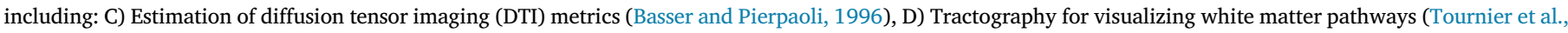

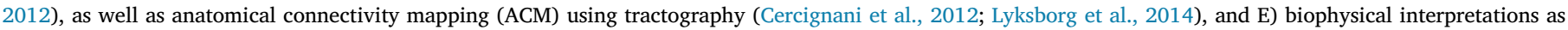

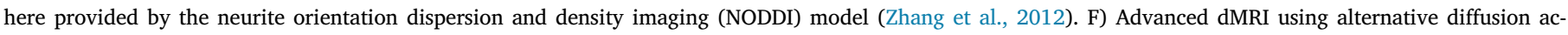

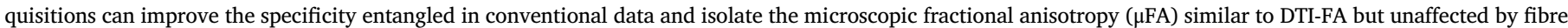

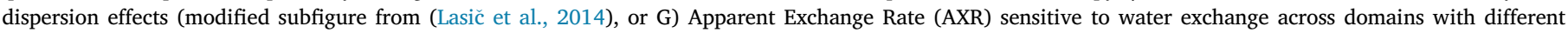
diffusivities (Nilsson et al., 2013).

such as cortical layers or long range brain connections. This widens the range of usable animal models from simpler cellular systems using nonvertebrate animals (e.g. Aplysia snail and squids - see review (Beaulieu, 2002)) and vertebrates with increasing brain volumes (e.g. rodents, ungulates and to non-human primates) or isolated brain slices or nerves (Kakkar et al., 2017; Flint et al., 2009). Fig. 3 shows examples of vertebrate animal brain models used in dMRI and the human brain.

In studies focused upon the gross anatomical organization or higher cognitive functions of the human brain, the monkey has traditionally been preferred because it is morphologically close to humans (Caminiti et al., 2009). The monkey brain is well described in terms of brain connectivity using non-invasive tracers and histology, i.e. staining of axons, myelin, cell types and their relation to physiology and brain function.
This makes the monkey brain unique as animal model for validation of tractography and microstructure, and due to its close anatomical relation which can easily be combined with human tractography and dissections (Innocenti et al., 2017; Hau et al., 2017; Alexander et al., 2010). Larger animal models such as pigs are increasingly used in neuroscience and disease models (Lind et al., 2007; Sorby-Adams et al., 2018). Fig. 3 shows that the pig brain has similar size as macaques, with a highly convoluted cortex. Furthermore, it provides a white matter complexity in terms of large fibre tracts that resemble the human brain, which makes the pig suitable for validation of tractography against multiple invasive neuronal tracers (Dyrby et al., 2007; Knösche et al., 2015). Smaller animal models like rodents are also widely used. Indeed their brain is small but they are easy to handle and are good models used for studying brain plasticity of 
A
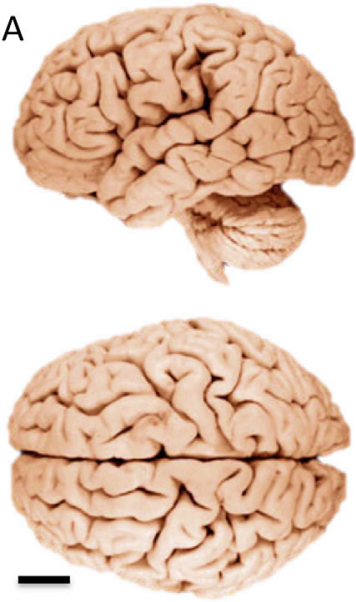

B
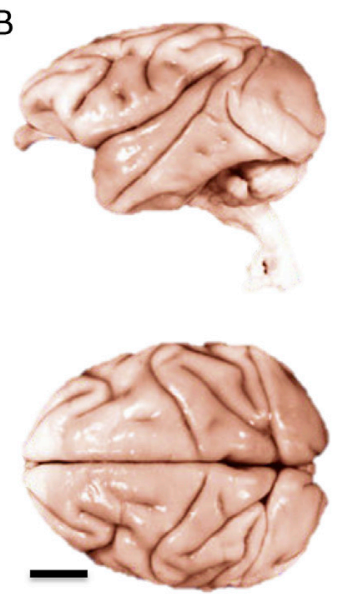

C
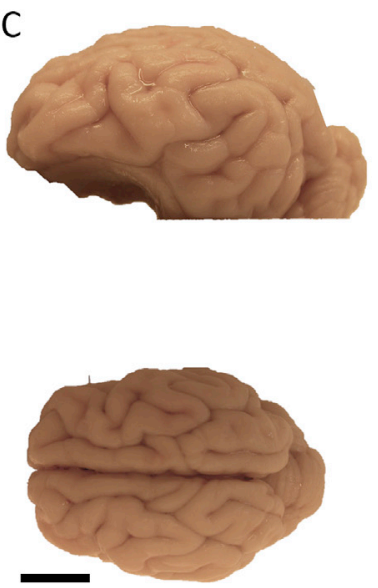

D
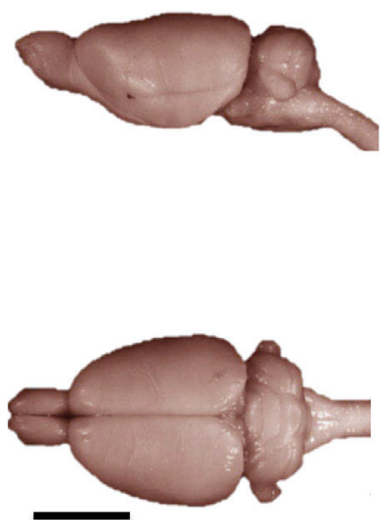

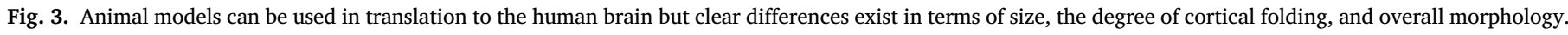

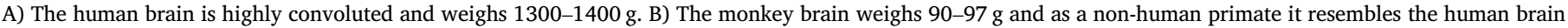

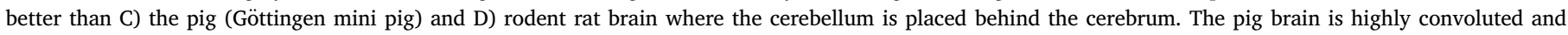

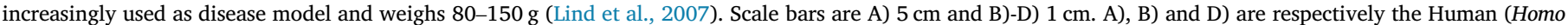

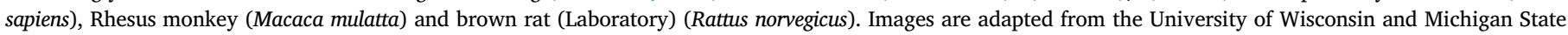

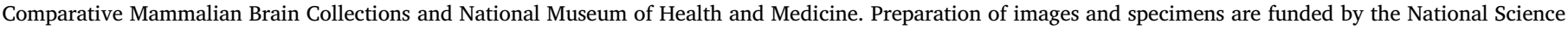

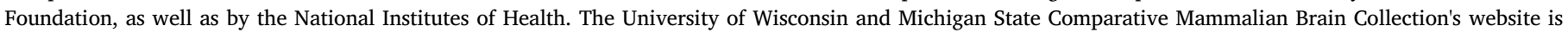
http://neurosciencelibrary.org/index.html.

microstructural features, e.g. the modelling of neurite density using dMRI and its application to detecting the impact of mild chronic stress on neurite density in cortical grey matter (Jespersen et al., 2010; Khan et al., 2016). Induced diseases, such as the experimental autoimmune encephalomyelitis (EAE) models, resemble pathologies in human MS (Budde et al., 2008). This provides a validation platform to both evaluate dMRI against histology as well as treatment effects. A different approach is the use of genetically modified knockout mouse models, e.g. of tuberous sclerosis neuropathology, to verify changes in microscopic anisotropy using dMRI (Kaden et al., 2016). Various demyelination animal models commonly used for dMRI validation are reviewed in (Edgar and Griffiths, 2009).

\section{Considerations for animal imaging}

The selection of the experimental setup for microstructural imaging is crucial to ensure the reliability of the MRI tissue contrast subject to validation. Most animal brains relevant for imaging research are several factors smaller than the human brain, and therefore the voxel size must be decreased to obtain similar anatomical information. Since voxel size is inversely related to signal to noise (SNR), small radio frequency (RF) coils designed for small animals as well as higher magnetic field strengths are used to ensure high SNR. SNR can also be increased by repeated measurements, but total scan time for living animals also has an upper limit. Therefore, it can be challenging to collect a rich preclinical in vivo data set for method validation of microstructure imaging, which both ensures sufficient SNR and provides the exploration of a set of acquisition parameters acquired at high-image resolution and with full-brain coverage.

The goal of ex vivo imaging is to attain a quality of the post-mortem tissue as close as possible to the in vivo state but without the limitations of physiological noise or time constraints. When the brain dies, the tissue starts a self-destruction process called autolysis. If not fixed immediately, the autolysis process will degrade the microstructural environment and already after the first 6-12 h, myelin sheaths start loosening around the axons (Hukkanen and Röyttä, 1987) as shown with electron microscopy

\footnotetext{
1 White Matter Microscopy Database: DOI 10.17605/OSF.IO/YP4QG | ARK c7605/osf.io/yp4qg and at Danish Research Centre for Magnetic Resonance: http://www.drcmr.dk/map-datasets.
}

(EM) in Fig. 4 (A-C). Autolysis can be slowed down by keeping the tissue at lower temperatures (e.g. $12{ }^{\circ} \mathrm{C}$ versus $24^{\circ} \mathrm{C}$ ) (Hukkanen and Röyttä, 1987). In animals, perfusion fixation should always be preferred when possible but one must be careful in preparing the fixative to ensure tissue quality (Cahill et al., 2012).

\section{Physical property differences between in vivo and ex vivo}

Successfully fixed tissue can be stored and rescanned for years but to ensure the collection of high quality ex vivo MRI data, the following must be considered (Dyrby et al., 2011):

- $\mathrm{T} 1$ and $\mathrm{T} 2$ relaxations parameters, which determine the signal decay of tissue types, are decreased. However, T2 relaxation, but not T1, can be partly prolonged by washing out the free fixative and thereby prevent loss of SNR (Dyrby et al., 2011; Shepherd et al., 2009; Leprince et al., 2015). Soaking brain tissue in gadolinium lowers T1 and enables shorter TR and faster acquisitions of e.g. diffusion MRI (D'Arceuil et al., 2007). However, the distribution of gadolinium in the extraand intracellular spaces is not known and may introduce a different weighting of the individual signal components. This effect should be considered in biophysical modelling and tractography.

- The T1 relaxation of grey matter is decreased ex vivo compared with white matter and the grey/white matter contrast may even be reversed in $\mathrm{T} 1$ weighted images as shown Fig. 4E versus Fig. 4F (Tovi and Ericsson, 1992).

- Diffusivity is decreased ex vivo, but not anisotropy (D'Arceuil et al., 2007; Sun et al., 2005). Therefore, the selected b-values for ex vivo dMRI should be approximately 3-4 times higher than in vivo to reflect similar levels of signal attenuation (D'Arceuil et al., 2007; Dyrby et al., 2011) (Fig. 4H). For example, in biophysical models like axon diameter estimates, the lowered diffusivity post-mortem is accounted for when designing the MRI protocols and analysing data (Alexander et al., 2010).

- Short-term instabilities are both a motion artifact and a temperature stabilization effect appearing within the first hours of scanning, as shown in Fig. 4D, and cannot easily be corrected. The source of the motion artifact is a mechanical process caused when larger tissue samples settles in the coil after handling and preparation (Dyrby et al., 2011). 


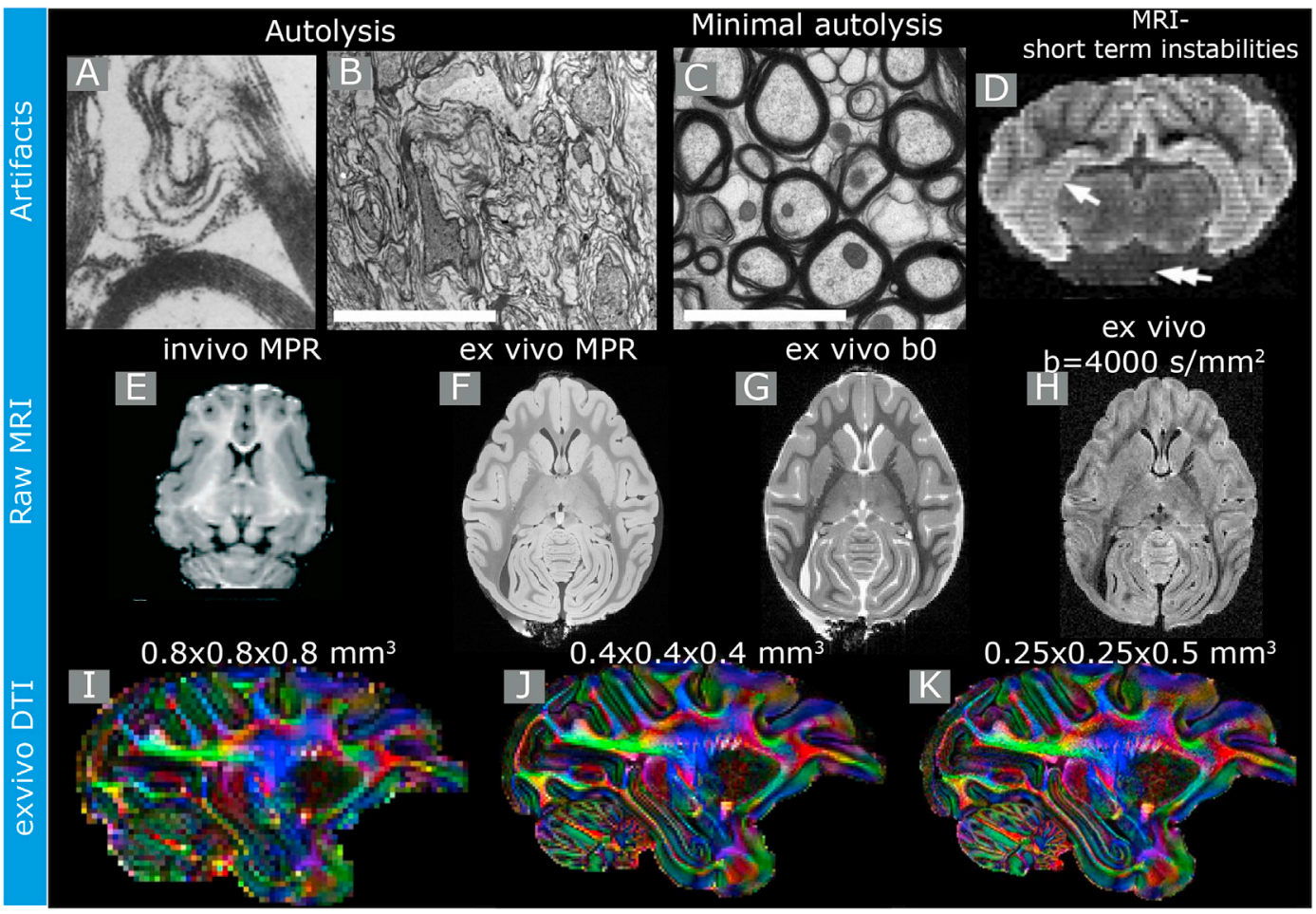

Fig. 4. Ex vivo diffusion MRI has the potential of creating high-quality MRI of intact fixed brain before preparing the tissue for histology, but one must be careful when trying to avoid artifacts from improper tissue preparation or scanning procedures. A) Autolysis, a tissue self-destructive process, is visible after $6 \mathrm{~h}$ on EM as loosening of myelin sheaths (modified from Fig. 2C in (Reprinted from ref. Hukkanen and Röyttä, 1987, Copyright, 1987; with permission from Elsevier) in x36,500). B) EM of a failed fixation process shows deformed axons and separated myelin sheath. C) EM of well perfusion fixed tissue shows clear, round axons wrapped with tight layers of myelin ((B) and C) courtesy of M. Burke, Howard University, and M. Ptito, University of Montreal. D) Short-term instabilities due to physical handling or temperature stability can be seen as stripes in a 2D spin-echo sequence with a $21 \mathrm{~h}$ time lag between even and odd slices (reprinted from Fig. 2 (Dyrby et al., 2011) with permission from John Wiley and Sons, Copyright 2010 Wiley-Liss, Inc.). Raw MRI of E) in vivo pig brain and ex vivo monkey brain using F) 3D T1 weighted magnetization-prepared rapid gradient-echo (MPRAGE), G) A T2w non-diffusion weighted image i.e. $\mathrm{b}=0 \mathrm{~s} / \mathrm{mm}^{2}$, and $\mathrm{H}$ ) a diffusion weighting of $\mathrm{b}=4000 \mathrm{~s} / \mathrm{mm}$ applied along a single direction using a preclinical 4.7 T Agilent MRI scanner (pig and monkey data sets can be downloaded ${ }^{1}$ courtesy of TB. Dyrby). Similar I-K) show examples of colour-coded FA maps of a fixed monkey brain acquired in various image resolutions using the ex vivo pipeline described in (reprinted from Dyrby et al., 2011, with permission from John Wiley and Sons, Copyright, 2010 Wiley-Liss, Inc.). Scale bar in B) and C) is $2 \mu \mathrm{m}$.

- Temperature controlled airflow around the tissue during ex vivo scanning is critical to ensure reliable dMRI data as diffusivity and relaxation times depend on temperature (Le Bihan et al., 1989). Heavy and varying diffusion weighting gradient use may cause significant temperature fluctuations of the scanner bore hence the sample.

- The shrinkage factor describe how much the ex vivo tissue volume is changed compared with in vivo. In microstructural imaging, it is essential for the translational argument using ex vivo tissue as a model for in vivo that the shrinkage factor is minimal and that the volume fractions of tissue compartments are similar, but it is still an open question. After perfusion fixation, the tissue shrinkage is reported to be approx. 5\% (Fox et al., 1985), but the shrinkage factor of tissue prepared for EM and light microscopy (LM), i.e. for axon diameter measurement, has been reported between $<1 \%$ and 65\% (Aboitiz et al., 1992; Lamantia and Rakic, 1990a; Riise and Pakkenberg, 2011; Houzel et al., 1994). The question to be answered is whether shrinkages mainly affect extracellular space, or if cell morphology is also affected (Hursh, 1939). In vivo measures of the fraction of the extracellular space suggest that it accounts for $15-36 \%$ of the tissue (Sykova and Nicholson, 2008) but visual inspection of EM shows almost a non-existing extracellular space between axons, as seen in Fig. 4C. The reason could be due to the tissue dehydration process needed before embedding tissue in epoxy resin for EM. However, it still needs to be investigated and quantified.

- Fixation stops all physiological functions of cells. Acute cell swelling effects following stroke or inflammation, easily detectable in vivo, are not guaranteed to have the same tissue contrast in ex vivo MRI. For example, the in vivo paramagnetic neuronal tracer manganese $\left(\mathrm{MnCl}_{2}\right)$ visualized hyper intense on T1w MRI is easily detectable in vivo but not ex vivo where it is released from intra-axonal transport and diffuses into the whole brain. Therefore, more research in the anatomical differences between in vivo and ex vivo is needed.

\section{Histological modalities - the quest for the gold standard}

The gold standard can be found within the histological techniques chosen according to the goals of the study, i.e. the stated microstructural MRI hypothesis. In this section we review selected invasive techniques that can be used to validate microstructural features at different anatomical length scales and image resolutions. Some validation techniques are classical whereas others are new but with potentials for microstructural MRI validation. We differentiate between classical staining techniques and neuronal tracing. The first shows micro- and mesostructural features, i.e. density, geometrical sizes, and shapes of cells, neurites and myelin, as well as their architecture. The second shows macrostructural features, i.e. the brain connections being both single or a bundle of axon trajectories. Finally, we comment on histological imaging techniques to display staining in terms of 3D field-of-view (FOV) and image resolutions.

\section{Classical staining techniques}

Nissl-like staining (cresyl violet, toluidine blue, hematoxylin-eosin, 
etc.) is often used to map the density of cell bodies in the brain.

The first histological technique to provide a detailed description of the cytology of the CNS was the Golgi silver staining. The technique is capricious and artifact-prone but it stains in detail individual neurons. This allowed Ramon y Cajal's monumental description of cell types in the CNS of several species (y Cajal, 1995). From this technique, little can be inferred on the connectivity of the neurons.

Staining of the fatty myelin wrapped around axons is often used in structure tensor imaging as information for validation of estimates of axonal orientational organization. Many different myelin staining techniques exist. One of the authors (GMI) has routinely used the Gallyas method.

Many more techniques exist for the staining of nervous tissue and some examples relevant for microstructural validation are shown in Fig. 5. It should be kept in mind that each histological technique is highly specific to a single microstructural feature as shown in Fig. 5 and may not reflect all features relevant for the dMRI contrast. However, they can be combined such as staining myelin and cells as shown in Fig. 5 (D, E) to provide a more complete image of the underlying true microstructural environment.

\section{Neuronal tracer techniques}

The long nagging issue of brain connectivity has advanced from degeneration methods (retrograde degeneration of neuronal cell bodies; axonal degeneration as with Marchi, Nauta, or Fink-Heimer methods) to the tracing of connections with axonally transported molecules, anterogradely, from the cell bodies to the terminations or retrogradely, from the terminations to the cell body. Neuroanatomical tract tracing techniques were masterly reviewed by (Vercelli et al., 2000; Heimer and
Robards, 1981; Zaborszky et al., 2006).

Modern anatomical tract-tracing techniques were initiated by the anterograde transport of radioactive amino acids, dating back to the discovery of axonal transport by Paul Weiss reviewed in (Brauckmann, 2004), which visualized axonal pathways and terminations via the activation of photographic emulsion (autoradiography). This approach turned out to be quite sensitive and produced a comprehensive picture of neural connections in the macaque brain, e.g. (Schmahmann and Pandya, 2006), but it could not resolve the individual axons or axon terminals. Nevertheless, the tracers spilled trans-neuronally across the lateral geniculate nucleus (LGN), which allowed the mapping of ocular dominance columns in the visual cortex (LeVay et al., 1980). Retrograde transport methods were initiated by the introduction of horseradish peroxidase (HRP; (Kristensson and Olsson, 1971)). This allowed a detailed visualization of the distribution of neurons at the origin of a connection, including their individual morphologies. In some applications, HRP, particularly when combined to wheat-germ-agglutinin (WGA), could be used for anterograde transport as well, although the resolution of the individual axons did not surpass the transport of amino acids. It was later discovered that neurons are rather undiscerning in what they actively pick-up and transport. Retrograde transport fluorescent molecules were introduced (Bentivoglio and Kuypers, 1982; Bentivoglio et al., 1979) as well as fluorescent microspheres of different colours (Katz et al., 1984) which were injected in separate targets to study axonal collateralizations (see also (Schain et al., 2014)). Because these tracers, unlike HRP, remained in cell bodies for a very long time without consequences for the neurons, they could also be used to take still frames of projections at different times, e.g. in the development of distinguishing between neuronal death and axonal elimination (Innocenti, 1981). The anterograde transport of paramagnetic metals $\left(\mathrm{MnCl}_{2}\right)$,
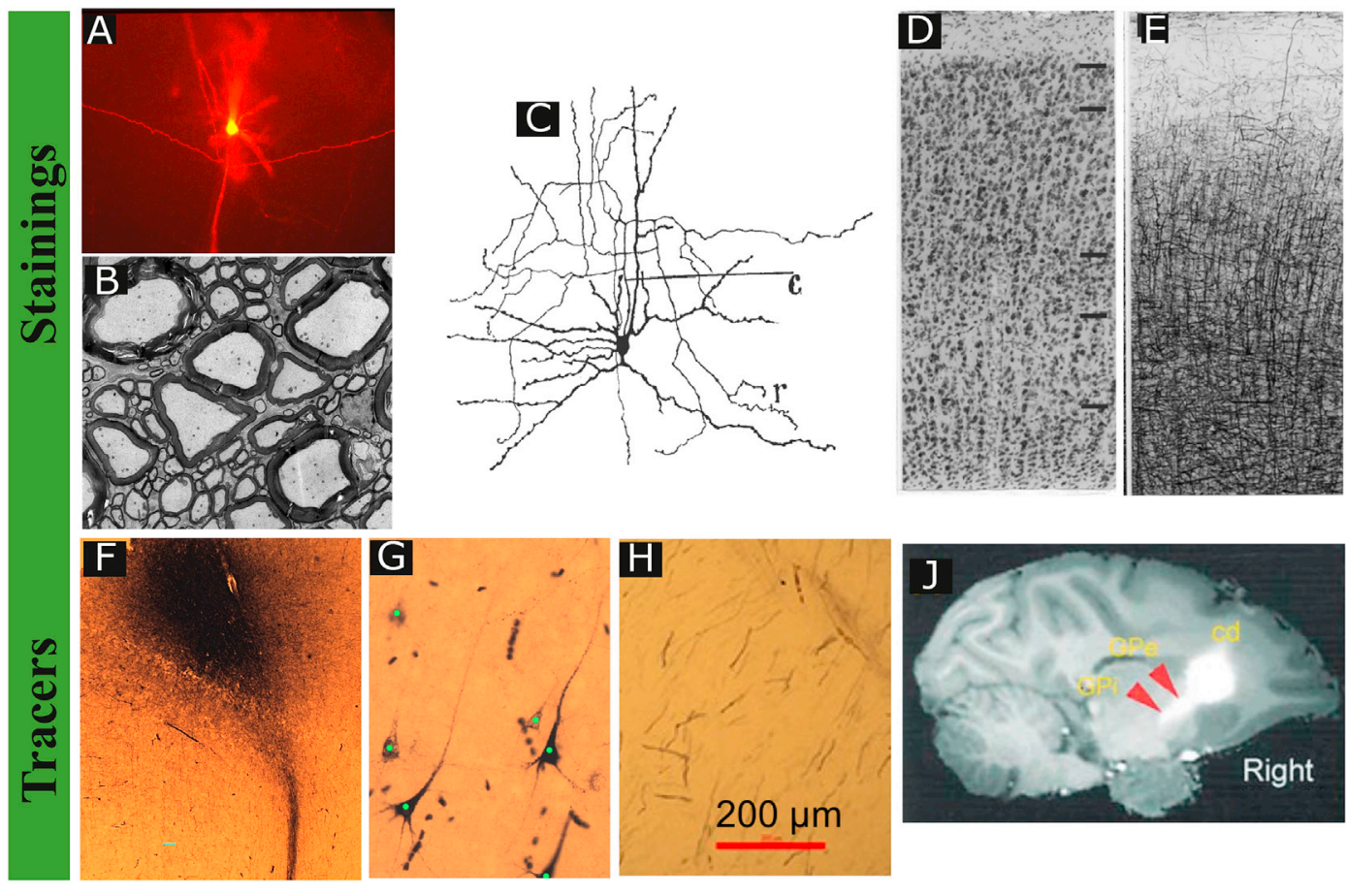

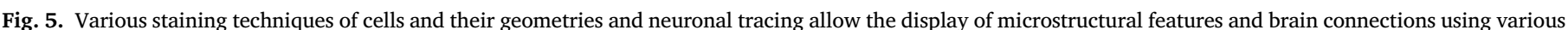

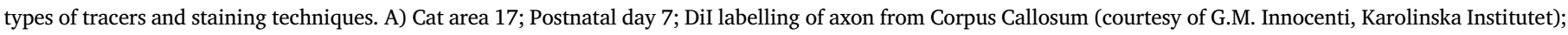

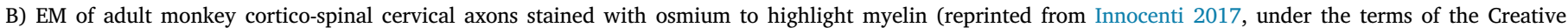

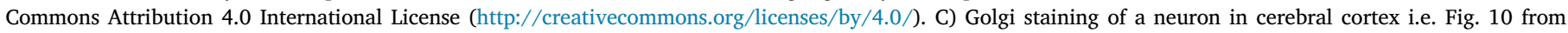

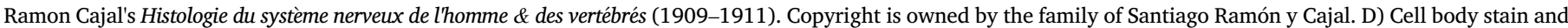

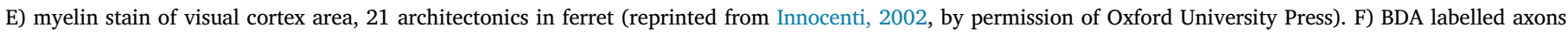

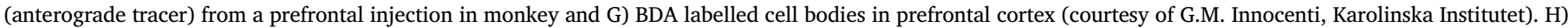

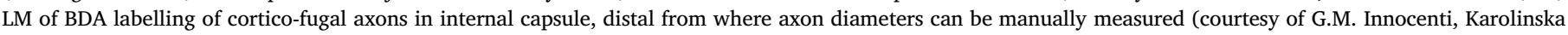

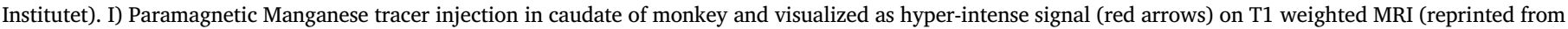
Fig. 5 in (Saleem et al., 2002), Copyright 2002, with permission from Cell Press). 
i.e. manganese, has the advantage of allowing direct MRI visualization of the intact living brain (same MRI space as tractography) albeit at the low image resolution of 3D T1 weighted MRI (Inoue et al., 2011) limits its use not to display individual axon trajectories but to larger brain connections. Opposite to other invasive tracers, manganese is measured in vivo. Because its signal is like a bolus signal reaching a maximum a few days after injection and then being washed out (Pautler, 2004), it can be challenging to set a common threshold for longer brain connection to be used for tractography validation (Knösche et al., 2015). Furthermore, it should be noticed that manganese has shown to have the unique feature of being a trans-synaptic tracer (Saleem et al., 2002). In contrast, the usage of anterograde transported dextran (Fritzsch and Wilm, 1990) or biocytin (King et al., 1989) obtained very detailed visualization of individual axons and axon terminals. In addition, lipophilic substances (DiI and DiO) can diffuse along lipid membranes in the dead brain (Honig and Hume, 1989) albeit slowly and with some difficulty in the adult brain. Other methods are finally answering the fundamental question of trans-synaptic connectivity (below) (Bentivoglio et al., 1979; LeVay, 1988).

Over the years, results obtained with the different tracer methods have been compared and have led to some insights of brain connectivity mapping, although the image is far from being complete, rarely quantitative and not straight-forward to generalizable across species. Nevertheless, important initiatives build structural connectivity mapping using tracers in e.g. monkeys (Schmahmann and Pandya, 2006; Bakker et al., 2012; Markov et al., 2012) and in mice (Oh et al., 2014).

Both retrograde and anterograde transport methods answer unequivocally the question of which brain structures are interconnected. In addition, the retrograde transport methods seem to imply the strength of the connection by disclosing the number of neurons involved in it, possibly to be translated into a number of streamlines (Markov et al., 2012; Donahue et al., 2016; van den Heuvel et al., 2015). This is at best a very first approximation. The strength of a connection, i.e. its power in activating a target, depends critically on the distribution of the terminal arbours (Tettoni et al., 1998) and on the size of synaptic boutons (Innocenti and Caminiti, 2017) and possibly on other factors, none of which is provided by the retrograde transport methods or easily achievable with current MRI methods. The anterograde transport of dextran or byocitin provides detailed visualization of single axons as shown in Fig. $5(\mathrm{~F}-\mathrm{H})$ as well as axonal arbours, including synaptic boutons. It provides a good gold standard for MRI studies aimed at microstructure, although it should be recalled that because of LM, resolution is mostly limited to myelinated axons $\geq 0.2 \mu \mathrm{m}$ in diameter.

\section{Imaging techniques: image resolution and field-of-view for 3D structures}

Histological validation techniques, as exemplified in Fig. 5, are often challenged by two factors:

1) Limited Field-of-view (FOV): Connectional neuroanatomy faces a major challenge in the transition from the meso to the micro or nano scale of neural visualization due to limited FOV and being represented as $2 \mathrm{D}$ histology slices.

2) Lack of effective image resolution: The finest anatomical structures that can impact diffusion cannot be imaged directly.

The effective image resolution limits, e.g. by LM, can to some extent be overcome by confocal microscopy as well as by other illumination methods such as super-resolution microscopy (reviewed in (Davis, 2009; Aoki, 2017)) taking advantage of progress in fluorescent microscopy. Nevertheless, the highest resolution still requires the use of EM and/or Scanning EM (SEM). Large FOV has been demonstrated by combining EM scanned slices aside into larger 2D planar image, but essential for dMRI is the volumetric composition, i.e. voxel size (reviewed in (Saliani et al., 2017)).

\section{D fluorescence microscopy imaging}

The fluorescent imaging methods has revealed new perspectives, in particular since it allows tracing neural pathways across synaptic connections, using genetically modified viruses, targeting the connections of specific cell types and many more (Beier et al., 2016; Martersteck et al., 2017). Two-photon microscopy imaging uses laser-scanning to scan in micrometre resolution the surface of a block of agar embedded fluorophore labelled tissue in micrometre resolution (Ragan et al., 2012). A 3D image volume with large FOV can then be generated by cutting away the previously scanned tissue section before a new section is scanned. Fig. 6A show a 3D-reconstructed whole-brain volume of tracer labelled axonal trajectories emanating from a single injection in the cortex of a mouse brain (Oh et al., 2014).

An alternative to two-photon microscopy is light-sheet fluorescence microscopy (LSFM) imaging that also uses a laser-scanning technique to scan an intact whole-brain volume, i.e. mouse or rat brain in micrometre image resolution. For whole-brain fluorescence imaging, LSFM requires the brain volume to be transparent so that emitted light from excited fluorophore labelled tissue substances can be imaged using a fluorescence microscopy. Different tissue clearance techniques exist, such as CLARITY and 3DISCO, etc., that wash out all lipids from tissue, making it transparent to light so that fluorescent labelled substances in the brain volume can be displayed (Silvestri et al., 2016; Richardson and Lichtman, 2015). LSMF have been used in combination with dMRI to study the effect of myelination and to its use for validation of DTI (Chang et al., 2017; Kamagata et al., 2016).

\section{D SEM and $X$-ray nanoscopic imaging}

Visualization of even smaller structures in 3D and their architecture, i.e. axons, dendrites and glial cells, as well as their subcellular components (synapses, spines, nuclei, and mitochondria, etc.), requires nanometre image resolution in large FOV which can be provided with imaging techniques such as SEM and synchrotron radiation micro-CT (SR $\mu \mathrm{CT}$ ), i.e. $\mathrm{X}$-ray. Kasthuri and colleagues used an automatic tape-collecting ultramicrotrome (AUTM) setup that continuously image and cut ultra-thin $30 \mathrm{~nm}$ slices of a tissue volume using SEM which afterwards were stacked into a large multiscale FOV 3D image volume (i.e. $2500 \times 2500$, $300 \times 300$ and $40 \times 40 \mu \mathrm{m})$. Fig. $6(\mathrm{~B}-\mathrm{C})$ show the segmented $3 \mathrm{D} \mathrm{EM}$ volume, and large parts of all neurons within a cortical column can be displaced down to the finest structural details as the synaptic cleft outlined between axonal terminals and the dendrites (Kasthuri et al., 2015).

Micro-CT with synchrotron radiation (SR $\mu \mathrm{CT}$ ) or X-ray microscopy is a relatively young discipline, as indicated in an early review by Fitzgerald et al. (Fitzgerald, 2000). A synchrotron radiation x-ray beam is highly collimated and several orders of magnitude more intense than that of the $\mathrm{x}$-ray tube. For ultra-high resolution x-ray imaging, enhancement by phase contrast is always present and enables 3D image reconstruction of intact sample volume. This is a great advantage in the imaging of samples with relatively homogeneous density, such as soft tissue samples (Beltran et al., 2011).

X-ray microscopy can in rough terms be divided into two categories: Full-field imaging and scanning probe imaging. In full-field imaging, the entire region of interest is illuminated and the x-ray wave-front transmitted through the sample is imaged with a camera (Bartels et al., 2015). In scanning probe $\mathrm{x}$-ray imaging, the $\mathrm{x}$-ray beam is focused upon a small spot and raster scanned across the field of interest to create an image. The latter method is slower but provides options for higher resolution well below isotropic $100 \mathrm{~nm}$, and provides multimodal imaging in terms of absorption spectroscopy, $\mathrm{x}$-ray fluorescence and diffraction as has been demonstrated in isolated sciatic nerve axon (Dučić et al., 2011). Fig. 6 (D and E) shows full-field $x$-ray phase contrast tomography with $100-300 \mu \mathrm{m}$ FOV and $50-100 \mathrm{~nm}$ image resolution, enabling 3D segmentation of individual axons, including their trajectories and fine

\footnotetext{
${ }^{2}$ http://connectivity.brain-map.org/projection.
} 

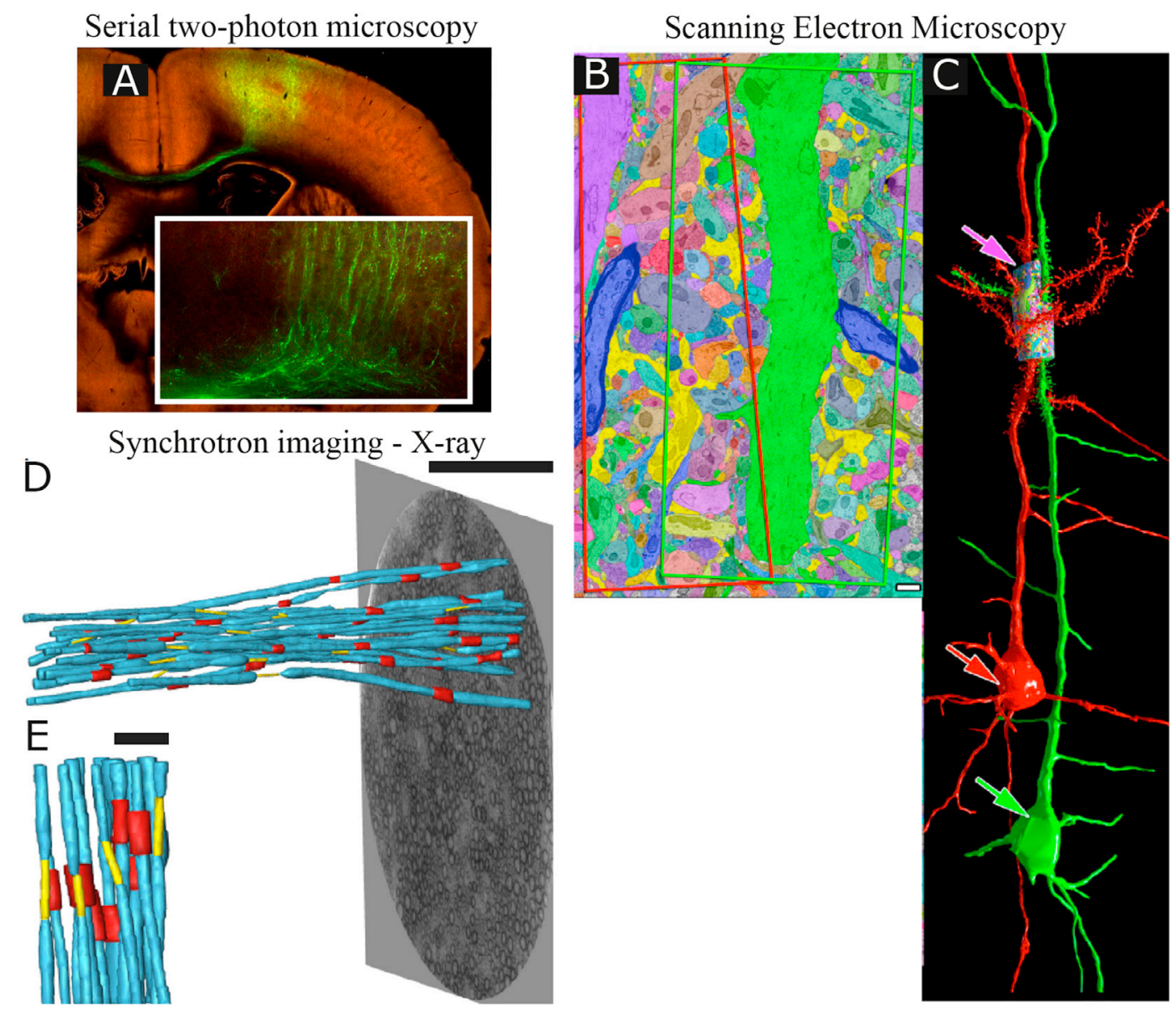

Fig. 6. Examples of three newer types of imaging techniques that enables 3D imaging of tissue microstructure with large FOV and in high image resolution. A) Neuronal tracing from primary motor cortex of mouse brain, using green fluorescent protein (EGFP)-expressing adeno-associated viral vectors that have imaged slicewise using high-throughput serial two-photon tomography (Image obtained online from the Allen Mouse Brain Connectivity online Atlas ${ }^{2}$ with an injection in Primary motor area (Oh et al., 2014). B-C) Multi-scaled SEM of 2D slices that were reconstructed into a 3D volume of neocortex. B) Manually thresholded segmentation of fine microstructural details, each with different colour. C) 3D surface rendering of two pyramidal cells in neocortex. The pink arrow indicates the place of belonging for the manual segmentation part in B) (B and C reprinted from Fig. 3 in (Kasthuri et al., 2015), Copyright 2015, with permission from Elsevier Inc.). D), and E) show the projection of 3D-rendered axons with i.e. their myelin sheaths (blue) and nodes of Ranvier (yellow), Schmidt-Lanterman incisures (red) obtained from synchrotron x-ray 3D data of intact nerve sample in isotropic $100 \mathrm{~nm}$ image resolution (from Fig. 8 in (Reprinted from Bartels et al., 2015, Copyright, 2015; Elsevier Ltd.). Scale bars are D) $100 \mu \mathrm{m}$ and E) $10 \mu \mathrm{m}$.

anatomical features (i.e. nodes of Ranvier). This type of x-ray microscopy can provide unique 3D information where intact nerves were used for validation of dMRI (Kakkar et al., 2017). Teh et al., used x-ray phase contrast in $3.6 \mu \mathrm{m}$ image resolution and combined with ex vivo dMRI to display cellular architecture of intact fixed rat heart (Teh et al., 2017).

\section{Validation of diffusion MRI techniques across anatomical length scales}

\section{Tractography validation (macrostructure)}

The many methodological challenges with tractography were already discussed in the first studies exploring the technique (Conturo et al., 1999; Mori et al., 1999). Conturo et al. used functional MRI (fMRI) to locate the lateral geniculate nucleus during visual stimulation and used this as a seed region and demonstrated its connections to the primary visual cortex. This shows an early example of an elegant cross-validation of tractography.

A number of excellent reviews already exist discussing the pitfalls of tractography, the attempts to validate the results, and perspectives for methodological improvements have been published (Assaf and Pasternak, 2008; Jbabdi and Johansen-Berg, 2011; Jones and Cercignani,
2010; Maier-Hein et al., 2017; Jeurissen et al., 2017). Therefore, only a few cornerstones will be recalled here, focusing in particular on work including histology and neuronal tracer data sets for validation.

Dyrby et al. (2007) demonstrated the ability to perform a direct validation between tractography and two simultaneously injected tracers in the same Göttingen minipig brain (Dyrby et al., 2007). The tracers included the anterograde axonally transported manganese $\left(\mathrm{MnCl}_{2}\right)$ visible with MRI and biotinylated dextran amine (BDA) used for histology as shown in Fig. 7 (A, B) (Dyrby et al., 2007). The tracers were single-sited injections in the somatosensory, motor and prefrontal cortex (SC, MC and PFC, respectively). The paramagnetic $\mathrm{MnCl}_{2}$ tracer allowed a 3D visualization of connections in vivo with a 3D T1-weighted MPRAGE sequence (Fig. 7D). Although the compromises of in vivo scan time versus sufficient SNR, the image resolution (isotropic $0.6 \mathrm{~mm}^{3}$ ) allowed visualization of tracer-labelled axonal bundles that tractography also reveals but not single axon labelled as the BDA tracer does. Multi-fiber tractography was performed in perfusion-fixed (paraformaldehyde) specimens, as shown in Fig. 7E, to ensure the high-quality diffusion MRI and image resolution (isotropic $0.5 \mathrm{~mm}^{3}$ ) compared with in vivo human from a whole-brain volume fraction relationship (Dyrby et al., 2011). Usually, but not always, the tracer and tractography methods agreed. Tractography revealed most of the same tracts albeit 

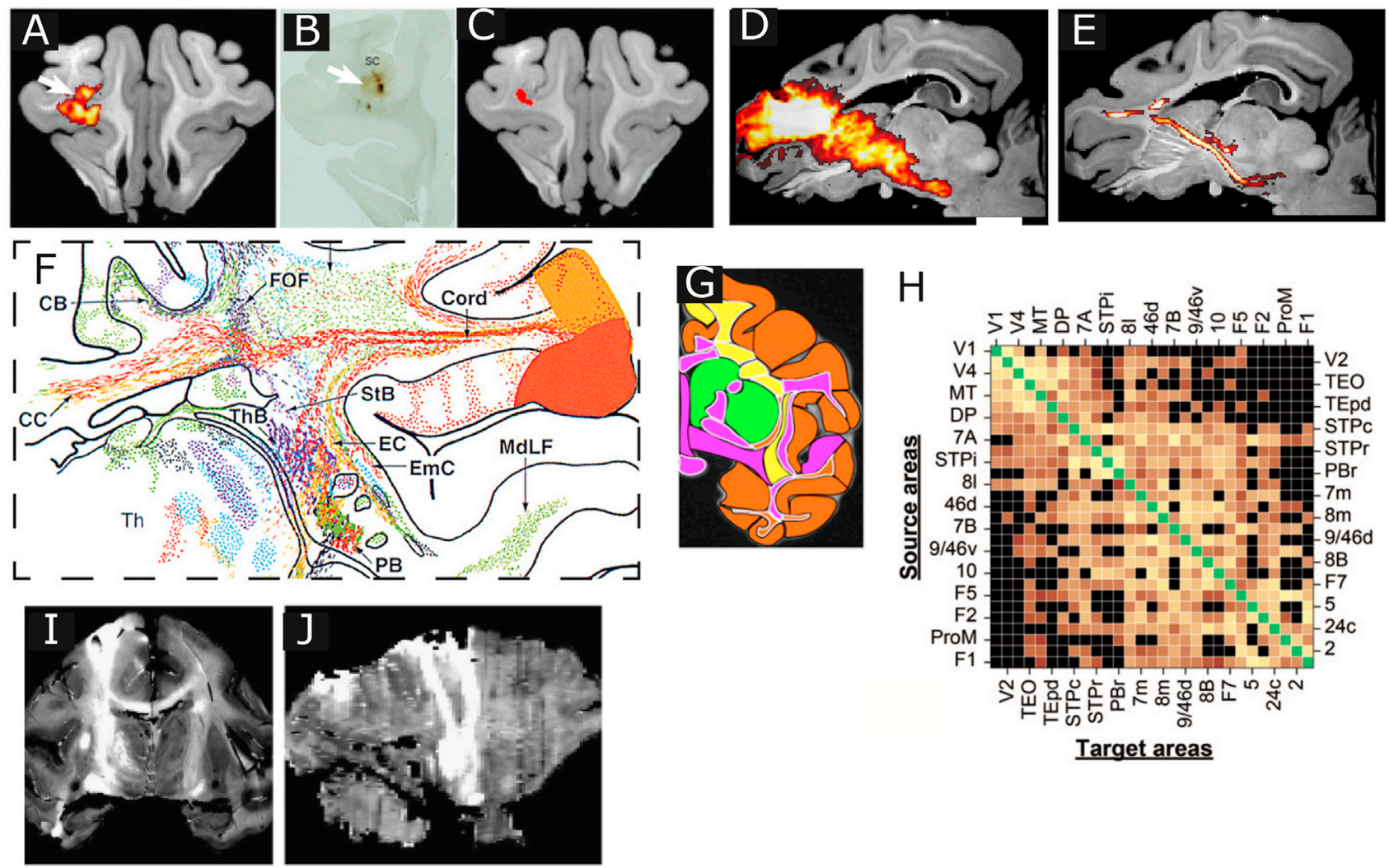

$\underline{\text { Target areas }}$

Fig. 7. Validation methods using invasive tracers for validation of tractography in various animal models. A-E) In pig brain: Single-sited injection with multi-tracer injection of anterograde (arrow) A) Manganese tracer visualized in 3D-MRI space and B) BDA tracer (visualized in a slice) used to define C) seed region for tractography (red region). D) Statistical t-map between baseline- and manganese T1W MPRAGE displays e.g. the cortico-nigral projection used to quantify the performance E) of ex vivo tractography on the same pig brains ${ }^{1}$ (modified from Figs. 1, 2 and 5 in (Reprinted from Dyrby et al., 2007, Copyright, 2007; with permission from Elsevier Inc.). F) In monkey brain: Injections in several brains of isotope labelled amino acids tracer analysed with dark-field microscopy. Slice-wise reconstruction of labelled axon trajectories is colour-coded according to their site of origin (modified from Figs. 6-13 in (Schmahmann and Pandya, 2006)). G) Tracer information from F) used to validate tractography (modified from Fig. 1 in (reprinted from Thomas et al., 2014). H) In monkey brain: Connectivity matrix produced from injections of fluorescent labelled retrograde tracers, fast blue (FsB) and diamidino yellow (DY). Labelled target neurons were counted and a structural connectivity matrix was generated and used in tractography studies, e.g. using ex vivo imaging (Donahue et al., 2016) (modified from Fig. 11 in (Markov et al., 2012)). In monkey brain: Five cortical injections of the WGA-HRP anterograde tracer in both the pre- and post-central gyrus. The tracer was visualized slice-wise, and reconstructed into a 3D volume to be validated against in vivo tractography (reprinted from Fig. 1 in (Dauguet et al., 2007), Copyright 2007, with permission from Elsevier Inc.).

with differences, including false negatives, e.g. the absence of cortico-thalamic projections from MC and PFC to the thalamus, and also false positives, e.g. projections from MC coursed around the thalamus, directly toward the brainstem. Importantly, Dyrby et al. (2011) also showed that tractography is robust in finding similar trajectories of brain connections across subjects but robustness, i.e. repetition, does not guarantee the validity. Dauguet et al. (2007) also demonstrated direct comparison in the same in vivo macaque brain, but acquired dMRI in only six gradient directions and in low image resolution that afterwards was interpolated from isotropic 1.5 to $0.75 \mathrm{~mm}^{3}$ voxels (Dauguet et al., 2007). DTI in vivo tractography was compared with a 3D reconstructed volume from 2D slices with the anterograde WGA-HRP histological tract tracer which is a highly challenging registration process as shown in Fig. 7 (I, J). The authors considered the influence of various parameters (FA, radius of curvature and step size) on the rate of false positives and negatives generated by tractography (Dauguet et al., 2007). Schmahmann et al., (2007) investigated association fiber projections revealed by Diffusion Spectrum Imaging (DSI) based tractography on ex vivo monkey brains and their tracer work obtained from Schmahmann and Pandya (2006) (Fig. 7F) and found a good concordance between the two techniques (Schmahmann et al., 2007).

These pioneering works were followed by a more systematic comparison of a whole brain connectivity matrix analysis that describes the correlation between how cortical regions are structurally connected to each other using tractography and axonally transported tracers in mice
(Calabrese et al., 2015) and in monkeys (van den Heuvel et al., 2015; Donahue et al., 2016) as well as comparisons of tracer and tractography on ex vivo monkey brains with in vivo human tractography (Jbabdi et al., 2013, Innocenti et al., 2017). Note that structural connectivity analysis between two methods does not describe the spatial agreement in the fibre trajectory between cortical regions but uses a connectivity matrix of e.g. neuronal labelling describing the correlation between source and target regions as shown in Fig. $6 \mathrm{H}$ from (Markov et al., 2012). The work in mice showed a correlation coefficient in structural connectivity between tracer and tractography of 0.42 , indicating a limited agreement between the two methods. True positive rate was $62 \%$ and false positive rate was $26 \%$. Calabrease et al. explained that: "Relative to neuronal tracer data, tractography tended to underestimate connectivity in the cortex and overestimate connectivity in midbrain and diencephalon" (Calabrese et al., 2015). Work in the monkey brain using the similar histological dataset but using slightly different methods of analysis provided correlations in the structural connectivity between 0.21 using the cocomac tracer database (Stephan et al., 2001) and 0.59 (van den Heuvel et al., 2015; Donahue et al., 2016) using the tracer connectivity matrix from (Markov et al., 2012) shown in Fig. 7H.

Knösche et al. (2015) returned to the $\mathrm{MnCl}_{2}$ injected minipigs dataset of Dyrby et al. (2007) to compare advantages and disadvantages of different tractography methods versus the ground truth fiber trajectory provided by the tracer. As the authors acknowledge, the visualization of this tracer is challenged by the low resolution afforded by the 3D T1w 
MPRAGE sequence compared with the size of axonal tracts. A broad spread out of $\mathrm{MnCl}_{2}$ around the targeted pathways makes it hard to set a common statistical threshold of the tracer map to outline the whole trajectory of long range projections for tractography validation. Therefore the authors reflected on whether their manganese gold standard tracer actually is more a "silver standard" with similar challenges regarding thresholding results as in probabilistic tractography (Knösche et al., 2015). Nevertheless, the results clearly stated that some tractography methods provided better results, but no method outperformed the other. Even so, the general limitation appears to be the same as all methods: it is not possible to achieve high sensitivity (i.e. few false negatives) and high specificity (i.e. few false positives) at the same time. This has been concluded by several groups who used tracer data in pig or monkey brains for validation, or realistically generated synthetic data based on in vivo human dMRI (Knösche et al., 2015; Thomas et al., 2014; Maier-Hein et al., 2017).

What are the reasons for these difficulties with today's tractography methods and how could they be overcome? One authoritative, albeit pessimistic, view presented by Thomas et al. is that: "We believe our results highlight an inherent limitation of tractography: inferring fibre direction information from a water diffusion displacement profile is fundamentally a complex, underdetermined inverse problem that cannot be solved." This was seconded by van der Heuvel et al. concluding: “... our results underscore the fact that tractography alone will not be sufficient to build an anatomically accurate map of the human brain connectome" (Thomas et al., 2014; van den Heuvel et al., 2015). Nevertheless, the first study provides possible avenues for the future but still needed to be answered: "Future improvements in the accuracy of diffusion tractography will require innovations in MRI hardware, sequence design, data acquisition strategies, and tractography algorithms" (Thomas et al., 2014).

Important anatomical features affecting tractography are the impact of gyral bias, i.e. how streamlines terminate in cortex and potential distance effects (Reveley et al., 2015; Liptrot et al., 2014; Donahue et al., 2016; Schilling et al., 2018).

\section{Neural connections obey to developmental rules}

We take the view that significant improvements might be the result of a better understanding of the "mistakes" tractography generates, relative to histology (Jbabdi and Johannes-Berg, 2011). Many methodological pitfalls must be considered in the acquisition as well as pre- and post-processing of dMRI data (Jones and Cercignani, 2010). Errors introduced by fiber crossings at specific brain sites are also well known in tractography analysis (Tournier et al., 2011; Behrens et al., 2007).

Possible solutions might be found in the developmental rules underlying brain connections, i.e. the growth of axons using guidance cues when establishing the brain network. In a loose sense, what tractography is trying to achieve is what development of connections achieves. Both tractography (streamlines) and maturating axons originats at one brain site and must then reach to the "correct" target, i.e. another brain site. The understanding of how this is achieved in the development is incomplete. Nevertheless, some principles are well established.

- Neurons differ in their genetic makeup, developmental history, and many aspects of their phenotype, including soma size, dendritic and axonal arbours, etc.

- Axons grow and elongate in the immature nervous system according to decisions made at the advancing tip of the axon, the growth cone (Goldberg, 2003).

- Growth cones read and follow contact guidance cues (guidepost cells, glia, other axons)

- Growth cones respond to attractive and repulsive signals.

- Growing axons compete for targets.

- Axonal projections are initially exuberant, that is, many projections are established in development, which are eliminated during later maturation (Innocenti and Price, 2005)

Some of the principles above are de facto used in tractography, in particular spatial information in the form of "inclusive" or "exclusive" ROIs seems to mimic the attractive (inclusion) and repulsive (exclusion) cues which guide growing axons. Others might improve the accuracy of tractography. Axons should grow orderly on anisotropic substrates. In development, this is provided by radial glia or by other axons. In their absence, axons, as streamlines, take abnormal trajectories (looping or grossly deviating from the bulk of axon/streamlines) which are nearly certainly artifacts to be eliminated (Fig. $8 \mathrm{~A}$ and C).

A recently proposed surface flow algorithm (St-Onge et al., 2015) seems to guide the growth of streamlines along an anisotropic substrate consisting of radial trajectories reminiscent of radial glia or corticofugal axons whose role in guiding developing axons has been documented

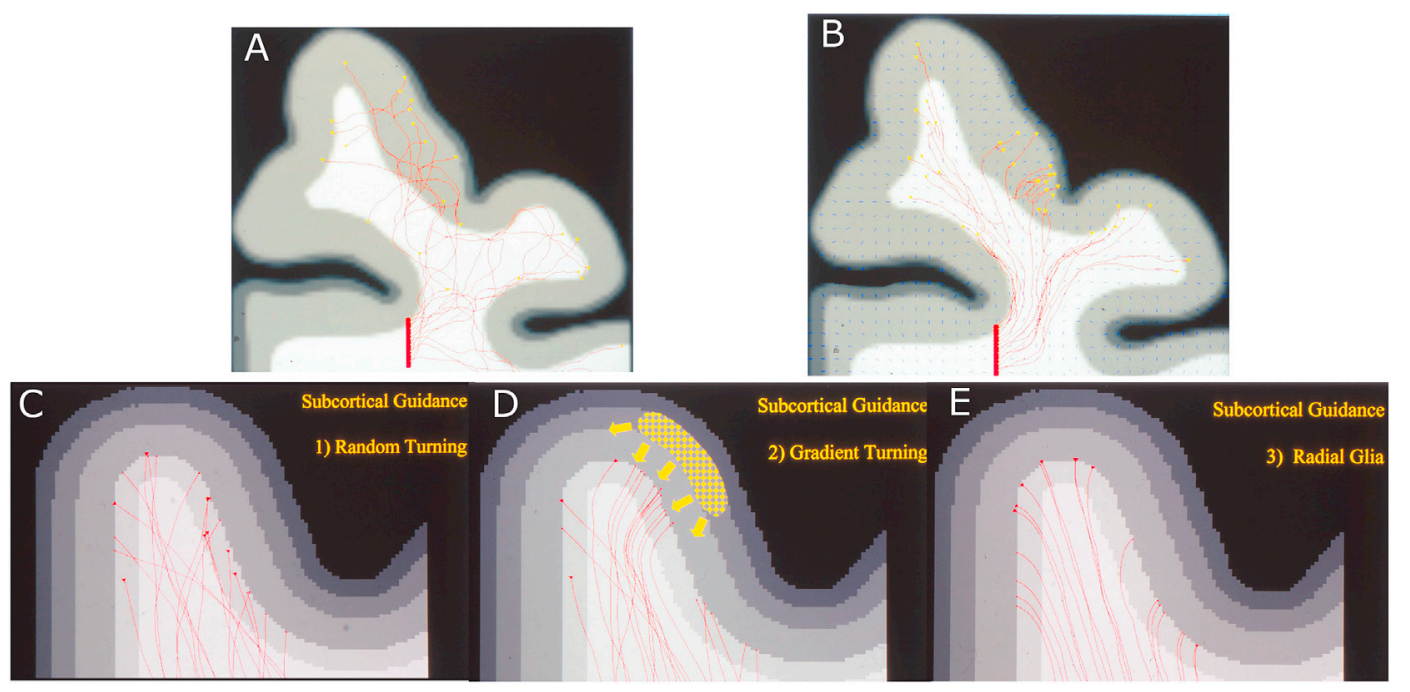

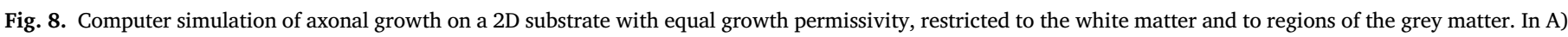

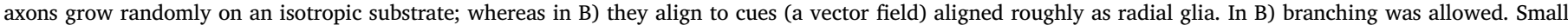

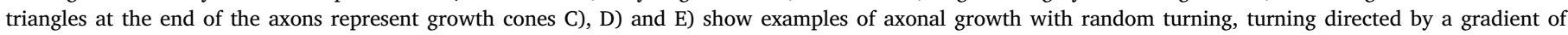

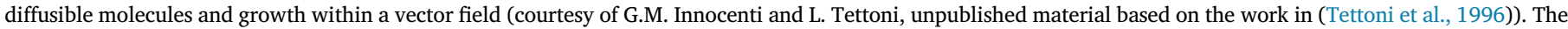
simulations mimic the development of callosal connections to areas 17 and 18 in the cat. 
(Norris and Kalil, 1991; Molnár et al., 1998). The elimination of false positives seems to be particularly difficult, when dealing with the human brain, in the absence of the reassuring histological information available for the monkey brain. In the developing brain, "exuberant" axonal projections are initially generated and later eliminated (Innocenti et al., 1977; Galea and Darian-Smith, 1995; Luo and O'Leary, 2005; Innocenti and Price, 2005; Innocenti et al., 2017). The elimination of the exuberant projections is under the control of functional criteria as proven by experimental manipulations which alter the normal selection process by altering brain function (reviewed in (Innocenti and Price, 2005); see also (Martin et al., 2004). We suggest that functional criteria could be used to accept or reject unexpected connections revealed by tractography. Functional criteria have been used to accept the existence of an interhemispheric parieto-striatal connection in humans which is less evident or absent in the monkey but which might be involved in language (Innocenti et al., 2017). Indeed, connections dealing with language are easier to accept in humans as they might be missing in primates (Rilling et al., 2011). The search for more general functional methods for the validation of tractography points to the use at least two techniques: i) voxel to voxel cross correlation in functional MRI (as discussed in Jbabdi and Johannes-Berg (2011) see (Chamberland et al., 2015); ii) dipole localization in high resolution EEG and EMG in combination with non-invasive brain stimulation techniques such as transcranial magnetic stimulation in healthy and diseased (Groppa et al., 2013; Fling et al., 2013). Each of these techniques has advantages and disadvantages however, we have chosen to skip a possible discussion regarding this due to its depth.

\section{Axon diameter measurements (microstructure)}

As seen in the validation of tractography, selecting a suitable validation strategy can be challenging, not only in relation to the reliability of the model being validated but also in relation to the validation data itself. This issue is no less acute for other models for dMRI. In the following, we focus upon non-invasive axon diameter mapping which is a research field that have faced challenges both in relation to questions regarding the biophysical modelling as well as interpretation of the validation setup. For insights into general biophysical modelling and practical applications of microstructural imaging with dMRI, see reviews (Assaf et al., 2013; Alexander et al., 2017).

Recent reports showing that neural connections, in particular cortical connections, are established by axons with different diameter (Caminiti et al., 2009; Tomasi et al., 2012; Innocenti et al., 2014; Innocenti et al., 2017) have opened a new field of investigations for at least four reasons:

1 Axon diameter correlates positively with conduction velocity (Hursh, 1939). This is one of the most robust structural-functional correlations in the neurosciences. Therefore, axon diameter and length provide a window on the dynamics of exchanges between brain sites. Axon diameter would also be proportional to firing frequency and could thus provide means for conveying different information (Perge et al., 2012).

2 Axon diameter is not necessarily static but a dynamic size. It may be modifiable during development and possibly in adulthood (reviewed in (Innocenti et al., 2016; LaMantia and Rakic, 1990b). Therefore, measuring axon diameters might provide a window on regressive pathologies of brain connectivity as well as on restoration of connections to be used in clinical settings. For example, degeneration of mainly the smallest axons has been reported in multiple sclerosis (MS) (DeLuca et al., 2004).

3 Axon diameter correlates with other morphological features of a connection. It has been known for long that it is proportional to the thickness of the myelin sheath and to the distance between Ranvier nodes (Ford et al., 2015). It has also been shown that it is also proportional to the size of the cell bodies of origin as well as to that of the synaptic boutons (Tomasi et al., 2012; Innocenti and Caminiti, 2017;
Cullheim and Ulfhake, 1979). While the morpho-functional correlate of the first is unclear, the second probably means that thicker axons also release larger amounts of neurotransmitters and therefore robustly impact the postsynaptic targets.

4 Microstructural imaging using MRI has opened the opportunity for axon diameter estimation non-invasively that allow us to follow the structural dynamics of the brain network and link it to physiology.

In the adult mammalian brain, axons thicker than roughly $0.2 \mu \mathrm{m}$ in diameter are myelinated. Therefore they express an inner diameter (d) and an outer diameter (D) with the g-ratio $(g=d / D)$. The g-ratio affects conduction velocity and is constrained to approximately 0.7 in most or all central nervous system (CNS) axons, a value justified by theoretical considerations (Rushton, 1951).

The adult mammalian brain also contains a number of unmyelinated axons, with diameters below $0.2 \mu \mathrm{m}$. The function of these axons is unknown. They fall below the resolution of conventional optical microscopy and the electrophysiological evidence of their existence is scanty to say the least. We have suggested that they might constitute a pool of axons to be myelinated under special circumstances, although this would be difficult to prove (see discussion in (Innocenti et al., 2016)). Fortunately, the number of unmyelinated axons seems to decrease in the larger brains. Therefore, they are not a crucial concern of studies aimed at investigating the human brain with diffusion MRI where the size of the smallest axons today cannot be estimated due to gradient hardware limitations and sequence designs (Dyrby et al., 2013).

The potential of non-invasive estimation of axon diameter using dMRI cannot be emphasized enough in the view of possible applications to studies of axonal properties in health and disease. Different basic biophysical models for axon diameter estimations have been introduced (Assaf et al. 2008, 2013; Stanisz et al., 1997; Alexander et al., 2010) and these can be extended to include non-axonal compartments (Panagiotaki et al., 2012).

Axon diameter estimations include both the biophysical model and acquisition setup to reduce scan time for application in living human brain, e.g. the ActiveAx framework (Alexander, 2008; Dyrby et al., 2013; Alexander et al., 2010). Firstly, it is important to state that histological methods are measuring axon diameters cross-sectionally whereas axon diameter estimations from dMRI are weighted by the intra axonal volume fraction because MRI is a volumetric measure (Alexander et al., 2010).

Data from EM of osmium stained axons that allow direct quantification of axon diameters and myelin thickness in the midsagittal plane of corpus callosum have been widely used in many validation studies (Fig. 9A). Aboitiz et al. (1992) and Lamantia and Rakic (1990a) provided histograms of axon diameter distributions in monkeys and humans (Fig. 9B) (Aboiti et al., 1992; Lamantia and Rakic, 1990). LM of either BDA labelled axons or myelin staining also provide axon diameter measures in monkeys and humans but with limitations in regards to quantifying the smallest axons (Riise and Pakkenberg, 2011; Innocenti et al., 2014). It should be noted that the BDA tracer labelling is not only used to measure axon diameter distributions of a brain connection but also for painstaking mapping of brain connections. The latter provides the first insights into a topological organization of conduction speed in the brain network (Innocenti et al., 2014; Innocenti et al., 2017).

Assaf et al. (2008) validated their AxCaliber method using ex vivo dMRI and against EM in the optic and sciatic nerves, and in the pig spinal cord. The axons in the spinal cord is a suitable region for method development as the axons are highly aligned, but collateral fibers branching off the main pathway for terminations in white matter could bias the estimates (Lundell et al., 2011). The AxCaliber assumes the true axon diameter distribution (ADD) to roughly agree with a Gamma distribution. Ex vivo dMRI analysis of optic nerve and sciatic nerve provided estimated mean axon diameters and ADD nearly overlap those obtained with EM completely as shown in Fig. 9D. They also reported that smaller axons $(<2 \mu \mathrm{m})$ could not be detected. However, the estimated mean ADD dMRI clustering in the spinal cord matched rather poorly with the 


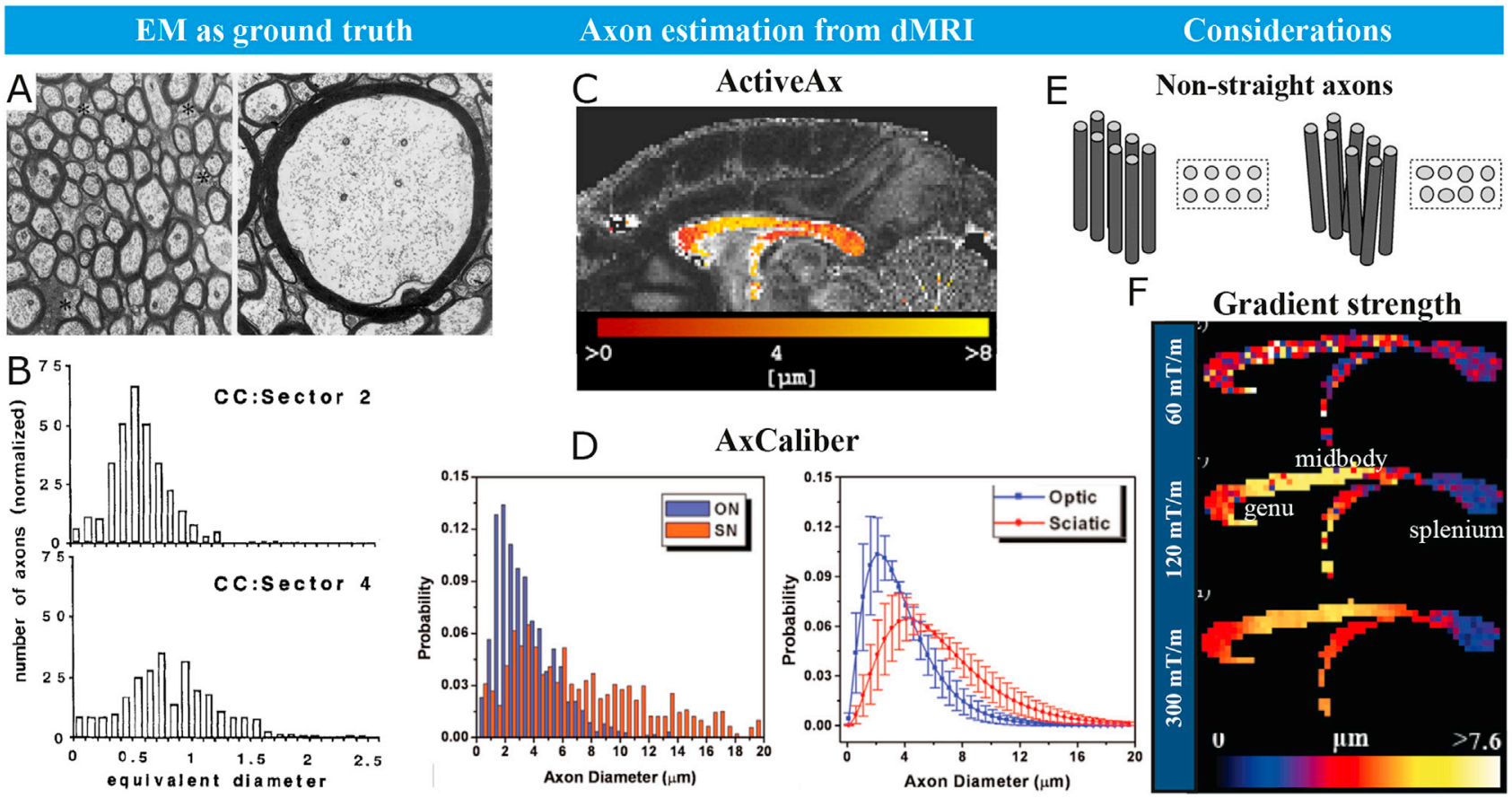

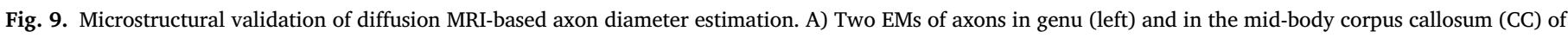

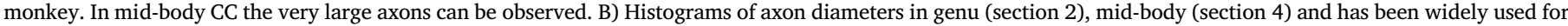

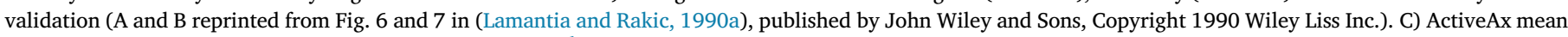

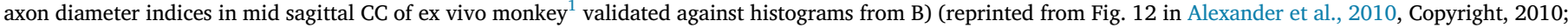

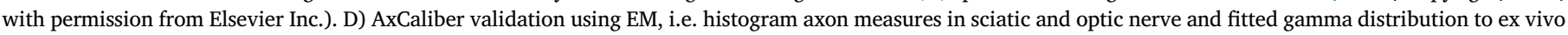

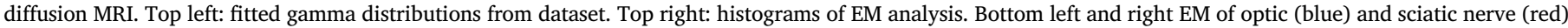

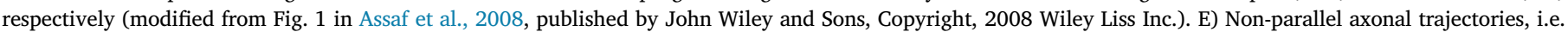

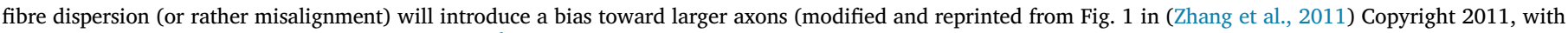

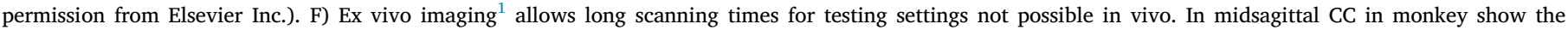

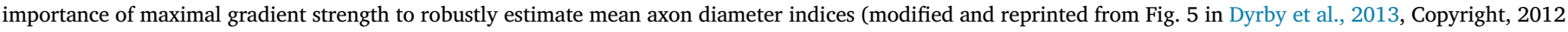
Copenhagen University Hospital Hvidovre. Magnetic Resonance in Medicine Published by Wiley Periodicals, Inc).

histological clustering (see Fig. 4 in (Assaf et al., 2008)).

The choice of the species and of the histological techniques remained an open question as the broadness of ADD varies across species with for instance a broader ADD in different primates (Caminiti et al., 2009), in different stages of maturation (Colello et al., 1994) and in normal aging (Aboitiz et al., 1996). However, the mean axon diameter peak show minor changes and is typically less than $1 \mu \mathrm{m}$ among many species or stages in life (Innocenti et al., 2014).

Alexander et al. (2010) introduced the ActiveAx which provides a more experimentally realistic measure: the axon diameter index rather than assuming a parametric distribution of axon diameters as in AxCaliber (Alexander et al., 2010; Dyrby et al., 2013). The ActiveAx axon diameter index corresponds to the mean axon diameter of the true ADD as found with EM but is volume weighted. It was named axon index to clarify that ActiveAx does not provide quantitative axon diameters but is an axon diameter weighted contrast that can be influenced by the choice of acquisition parameters and model assumptions (Dyrby et al., 2013). The method was compared with the mean axon diameter typically obtained from the axon diameter histograms in CC provided by (Lamantia and Rakic, 1990a; Aboiti et al., 1992) in monkeys (shown in Fig. 9B) and humans, and have been applied both in vivo and ex vivo on monkey, human and mouse brains (Alexander et al., 2010; Dyrby et al., 2013; Sepehrband et al., 2016). ActiveAx restituted in corpus callosum (CC) the regional difference in axon diameters as expected with EM, but, similar to AxCaliber, provided larger indexes of mean axon diameter than those observed with histology (see Fig. 9 in Alexander et al., 2010) (Alexander et al., 2010; Dyrby et al., 2013; Sepehrband et al., 2016).

The difference between the histological and the dMRI data is interesting because the constant differences between the two estimates suggest that the dMRI data does provide an axon diameter weighted image contrast. However, there might be a discrepancy between what we think and what we actually are estimating of the ground truth. Here, simulation experiments which use histological data to generate realistic synthetic axon substrates have proven to be a powerful tool for getting new insights into the function of biophysical models. For example, histograms have been used for demonstrating the existence of a sensitivity profile for dMRI sequences where the smallest axons do not provide signal alterations larger than realistic noise levels and the used diffusion sequence (i.e. lower-bound of $\sim 2.5 \mu \mathrm{m}$ depending on the applicable gradient strength and waveform) (Alexander et al., 2010; Dyrby et al., 2013; Nilsson et al., 2017; Sepehrband et al., 2016).

The existence of a lower-bound in the dMRI estimate of axon diameters has given rise to discussions in the literature and highlights the challenges in applying cross-disciplinary validation of MRI techniques against well-established histological literature (see (Horowitz et al., 2014) vs (Innocenti et al., 2015)). Similarly, Benjamini et al. (2016) introduced an interesting approach for non-parametric ADD estimation, using a double diffusion encoding (DDE) sequence (Benjamini et al., 2016). The validation included EM of the spinal cord of the ferret. Although the distributions of histological and axon diameter estimates overlap (see Fig. 6 in Benjamini et al., 2016), the histograms of EM diameters are shifted to values considerably larger than those found in the monkey (Firmin et al., 2014). It is not straightforward to say what is right and what is wrong, as no independent literature on ADD in ferret spinal cord seems to exist, and this underlines the relevance of considering a validation strategy that uses an animal model and/or an anatomical region where additional independent anatomical data exist, e.g. from other groups. 
The question about biases in axon diameter estimations using dMRI in relation to the ground truth is generally still a burning question simply because what is believed to be the ground truth as the i.e. EM or LM have limitations as well. For example:

I Why are axon diameter estimations from dMRI typically overestimated compared with histology? Is it due to the unknown tissue shrinkage effect reported in literature possibly caused by the tissue dehydration process prior to histology? If so, are both the intra- and extracellular compartments equally shrunk as typically assumed in modelling (Alexander et al., 2010; Sepehrband et al., 2016) or is shrinkage only related to the extracellular space which in EM appears mostly non-existing (Fig. 4C, Fig. 5B and Fig. 9A), as discussed in a previous section? Axon diameter overestimation can also come from fundamental methodological mismatch since EM is showing cross-sectional 2D images whereas MRI generates axon diameter estimation within 3D volumes, i.e. voxels. The axonal cross sectional area will appear increased if not measured perpendicular to the axons, but noted that this scales as $1 /$ cos (angle) which is very small up to reasonable angles compared to the reported overestimations. Several dMRI studies suggest that axons are not guaranteed to form straight bundles, which can also cause overestimation of axon diameters (Zhang et al., 2011; Zhang et al., 2011; Nilsson et al., 2011; Dyrby et al., 2014a) as illustrated in Fig. 9F).

II Simulations on synthetic substrates mimicking ADD and analytical considerations show the existence of a lower-bound of detectable diameters (Dyrby et al., 2013; Nilsson et al., 2017). The lower-bound is higher than the main peak of histological axon diameter measurements (also after volume-weighted correction and possible shrinkage factor correction), but interestingly, the estimated axon diameter index shows an axon diameter contrast that agrees with histology. The difference between estimated sizes and histology may be explained by the simulation results suggesting that the smallest axon diameters cannot be detected and hence the major contribution to the dMRI signal in tissue is from the largest axons which will result in an overestimation. Further, exploring the information content in dMRI data and combining it with other qMRI methods could provide exciting avenues for extracting relevant biomarkers related to axon diameter (Novikov et al., 2018).

\section{Fiber dispersion effect (mesostructure)}

Investigating the mesoscopic scale between the macroscopic scale of the trajectories of axonal pathways and the microscopic features such as axon diameter is of major interest for several reasons. As discussed, one challenge in tractography is e.g. the bending, kissing and crossing fiber configurations that can result in the same signatures in the dMRI data but relate to very different connectivities (Tournier et al., 2011). The effects of mesoscopic organization will also be reflected differently in data acquired at increasing resolutions and might not improve fibre complexity (Schilling et al., 2017). This effect may partly be resolved by doing interpolation down to smaller voxel sizes as was validated using dMRI as its own gold standard when acquired at higher image resolutions (Dyrby et al., 2014b). Furthermore, the sub voxel orientational arrangement biases quantitative measures from DTI as well as biophysical modelling, e.g. axon diameter, indices and could have physiological relevance and thus be of interest as a measure of its own (Jespersen et al., 2012; Zhang et al., 2012).

The validation of those aspects could be a direct approach with single axon tractography that would image both diffusion and the axonal configuration simultaneously. Flint et al. demonstrated this on small spinal cord samples at ultra-high field with direct comparison to the same axons visible with LM (Flint et al., 2010). However, those extreme conditions are not yet possible on whole tissue samples and diffusion metrics may not be directly comparable at mesoscopic resolutions due to less invasive post mortem tissue preparations. Other, more indirect, comparisons to histology are therefore sought after. Leergaard et al., 2010, proposed a method for validating fiber orientation distribution (FOD) from dMRI with myelin derived FOD (Leergaard et al., 2010). Later, Budde and Frank proposed the structure tensor analysis based on spatial derivatives of 2D microscopy data (Budde and Frank, 2012). The structure tensor analysis provides information on the local directional organization in histological images. The input data could be any image data with histological preparation reflecting the microstructural features of interest, e.g. myelin maps for investigating the distribution of myelinated axons. Fig. 10 outlines the principles of structure tensor. The main value of this approach is the insight from the distribution of eigenvectors which through a local diffusion model can be related to the signal from a larger region with dispersion. However, the structure tensor eigenvalues are not proportional to those of the diffusion tensor. It is also important to realise that the structure tensor data reflect the structural data visible in the microscopy images of the histological preparation and possibly not all spaces relevant for water diffusion.

The 2D model was later expanded to 3D by Khan et al. (2015). This is in principle easy given an unbiased dataset. However, a practical challenge lies in acquiring microscopic 3D data and to understand and correct for artifacts in particular introduced by the image's point spread function (PSF) in the slice direction. Khan et al. approached this with confocal microscopy of $\sim 0.1 \mathrm{~mm}$ slabs that were later stitched together and corrected with the estimated PSFs. The effective image resolution was in the order of $0.4 \mu \mathrm{m}$ in plan and $1.2-2 \mu \mathrm{m}$ in the slice direction, depending on the depth from the surface. The structural eigenvectors from this dataset were compared to high resolution DTI with good agreement in a hippocampal slice from a rhesus monkey. Similarly, Salo et al. used a block-face SEM technique to produce 3D structure tensor data from the injured rat brain (Salo et al., 2018).
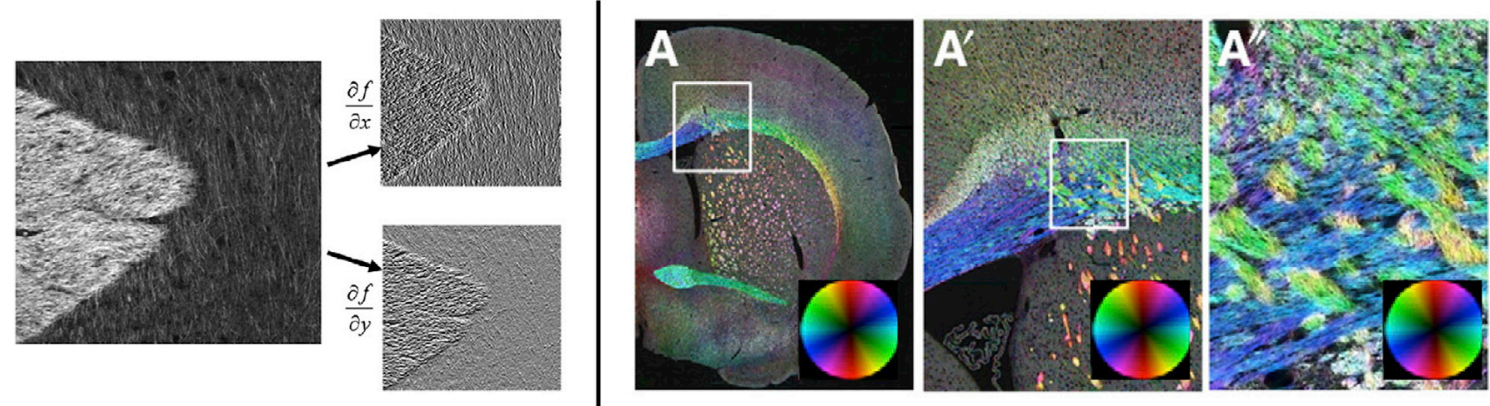

Fig. 10. Left: Structure tensor analysis uses spatial derivatives of histological images (here with a lipophilic dye (Dil) giving low light transmission in regions with high myelin content) to identify the orientational distribution at a certain resolution. Right: Structure tensor analysis in one coronal slice in the rat brain at different magnifications. Interwoven crossing fiber regions between callosal (blue) and ascending (green) fibers are seen in the highest magnification in $\mathrm{A}^{\prime}$ (Note that the colourcoding is in plane only and different from conventions used in DTI). Modified and reprinted from Fig. 1 and 2 in (Budde and Frank, 2012), Copyright 2012, with permission from Elsevier. 


\section{What we see is what we get}

Neuroimaging dMRI data analysis is mostly centred around neuronal interpretations but also non-neuronal cell types and tissue spaces are reflected in the measured data (see review (Alexander et al., 2017)). Understanding the contribution from non-neuronal components is therefore relevant. It is commonly reported that for instance lowered FA in white matter can be related to changes to the intrinsic qualities of axons, but non-neuronal sources of anisotropy could also be of relevance. One example was shown by Schwartz et al. investigating the aftermath of a spinal cord trauma model in rats (Schwartz et al., 2005). Here, the authors found increases in radial diffusivities in white matter as often reported following neuronal degeneration, but histological staining of not only the neuronal compartment suggested that radial glial scar tissue following the lesion created anisotropic structures perpendicular to the axonal direction and thus contributing to the lowered FA. In a more recent example of post mortem dMRI of human spinal cord samples with validation through structure tensor analysis of different cellular components were also considered (Grussu et al., 2017). This study showed indeed a strong correlation to neurite dispersion measures derived from dMRI and the 2D structural tensor analysis of the neuronal components. Furthermore, colocalized reductions in dispersion in both modalities were found in tissue affected by MS lesions. However, the dilution from other anisotropic structures such as gliosis could not be fully discarded in the neurite dispersion indices derived from dMRI.

A more direct comparison between histological stains reflecting cell types and the diffusion signal is possible with diffusion weighted spectroscopy (DWS) that allows in particular isolation of the intracellular neuronal and astrocyte signals from the NAA and Cho metabolite signals (Palombo et al., 2017). Ronen et al. compared 2D structure tensor data from human corpus callosum samples with dispersion models for the DWS signal (Ronen et al., 2013). They showed that microscopic dispersion played a large role in the neuronal signal, with local deviations of up to $15^{\circ}$ even in a well-aligned structure like corpus callosum. Furthermore, they showed larger dispersion in astrocyte fibrous structures and proposed a small contribution from their spherical cell body. Palombo et al. went one step further than the structure tensor analysis and used DWS data acquired over a large range of diffusion times up to $2 \mathrm{~s}$ to evaluate not only the dispersion of fibers but also the effects of length and branching structures of different cell types evaluated with Monte Carlo simulations in monkeys and mice (Palombo et al., 2016). This data showed good agreement to the overall astrocyte appearance segmented from confocal microscopy of cell specific stains with agreement to the difference across the two species.

Even though many effects can be predicted through forward modelling, heterogeneity poses a challenge when for instance dispersion of anisotropic structures and variation in sizes can give identical signals in the diffusion data. To solve this ambiguity, multidimensional diffusion encoding (MDE) has been proposed to provide a direct contrast to those two effects (Shemesh et al., 2016; Topgaard, 2017). MDE applies diffusion encoding in single or multiple directions within the same image readout which separates the effects of variations from differently oriented anisotropic tensors or distributions of different local mean diffusivities. Structure tensor analysis was used by Szczepankiewicz et al. in this context to evaluate dispersion and heterogeneity in tumour biopsies from humans (Szczepankiewicz et al., 2015). This demonstrated local anisotropic structures, entangled in FA but resolvable with $\mu \mathrm{FA}$, equivalent to the average local microscopic FA in the sample unaffected by dispersion (example shown in Fig. 2). Other novel MDE approaches incorporating sensitivity to diffusion time dependence in the short time domain can in contrast capture isotropic features, e.g. the dense granular spherical cell body layer of the cerebellum (Lundell et al., 2017). Those features have independently been captured with oscillating gradient spin echo techniques and correlates well with Nissl cell body stains (Aggarwal et al., 2012; Lundell et al., 2015).

\section{Concluding remarks and future perspectives}

We have reviewed the current literature intersecting dMRI, its indirect interpretations across different anatomical length scales, and the use of validation techniques that often provide direct insights into anatomy. It is the relatively low image resolution of dMRI that causes anatomical features across length scales to be intermingled, and the challenge is to disentangle them correctly. High image quality, high SNR, strong gradients and a wide range of parameters can be achieved using ex vivo imaging. Indeed, ex vivo imaging is also believed to be as good a structural model of in vivo but it has its pros and cons as we have highlighted. We have mainly focused on dMRI methods targeting axonal configurations from macroscopic to microscopic length scales. This research field is a good demonstration of how validation is the key to releasing its potential in clinical applications and biomedical research. In this process, we do not only get new tools; we also extend our knowledge about the ground truth. Changes in our knowledge may lead to changes in the underlying methodological assumptions, and hence the methodology itself. When that process has taken place, the methodological evolution has taken one step forward. We argue that this methodological evolution is an iterative optimisation that takes us through the following steps:

Loop until our method's results agree with gold standard, and/or until the updated knowledge of the ground truth can explain the discrepancies observed:

(i) Theory; Establish a methodology (e.g. mathematical model and/ or acquisition of diffusion) that links to a physical feature (e.g. anatomical geometry) based on current knowhow of the ground truth.

(ii) Implementation and realisation; Considerations of the interaction between the methodology and the measuring technique to be used in i.e. MR. Creating test data sets to verify the methodology, either by using computer simulations on synthetic generated phantoms, or by emulation where MR data is collected on a physical phantom.

Reflections regarding the experimental outcome: Describe unexpected deviations. Quantify the size of the effect in relation to different sources of noise for a given experimental setup. Consider updating the assumptions and/or the methodology. Consider going back to (i).

(iii) Validation; select one or several independent image modalities as gold standard(s) for validating the new method in a realistic setting, i.e. tissue environment (in vivo and/or ex vivo).

Reflections regarding the experimental outcome: Describe and understand deviations in relation to validation method(s). Adjust current know-how of the ground truth, the underlying assumptions and the methodology. Go back to (i) or (ii).

In the implementation and realisation step, simulations on synthetic phantoms have proven to be an important step along the way to investigate the parameter range accessible, fitting procedures and the level of method complexity that is supported by a given acquisition dMRI setup. It allows exploring isolated anatomical features in a deliberately simplified environment if direct validation experiments in real tissue are hard to generate. Therefore, as we reviewed, the simulation and validation steps are closely linked together as an efficient way of adjusting and updating current know-how and verifying the assumptions used. Simulations have been key in the verification of multi-fiber reconstruction methods for tractography (Alexander, 2005), in identifying the lower-bound in axon diameter estimates (Alexander et al., 2010; Dyrby et al., 2013), understanding effects in the complex extra-axonal space (Novikov and Kiselev, 2010) and recently in tractography by Maier-Hein et al. in tractography ISMRM 20015 challenge (Maier-Hein et al., 2017). The latter study used a realistic synthetic human brain dMRI data set 
generated from known brain connections that identified "bottleneck" regions and demonstrated their contributions to the general well-known false-positive problem in modern tractography methods.

The validation step is the most painstaking and time demanding step. It uses independent imaging technologies (e.g. histology) to provide an independent, either direct or indirect, view of the anatomy assumed to be mapped by the methodology in validation, i.e. dMRI. One practical challenge, as reviewed here, is that MRI is a volumetric technique with voxel sizes in the order of cubic millimetres. Therefore, each voxel contain an average of anatomical features at different length scales such as the mesostructural, i.e. non-straight axons (fibre dispersion, crossing, etc.) and microstructural, i.e. geometrical neuronal sizes, shapes and densities. If the anatomical multi length scale is not accounted for in the validation (maybe due to limited knowledge) then a discrepancy will be generated, waiting to be explained. One classical example is the impact of crossing fibres on DTI-based indices (direction and FA) used in tractography and microstructural clinical interpretations. Today, crossing-fibre models are standardly used in tractography and subsequently considered in biophysical modelling. However, clinically, many studies still report group changes in DTI without being fully aware that the underlying anatomy might be a mixture of meso- and microstructural effects, and not only microstructure. Further insights into microstructural organization using DTI can be obtained using Westins metrics (Westin et al., 2002). However, promising new clinically applicable dMRI sequences provide microstructural $(\mu)$ FA mapping where the unwanted mesotructural fiber effect has been reduced at the data acquisition step (Jespersen et al., 2013; Lasič et al., 2014; Yang et al., 2018). Efficient knowledge transfer of such new technologies between the technical and the clinical neuroscience areas is therefore wanted.

Future perspective is based on the know-how obtained in microstructural imaging and there is a tendency in increasingly complex models with the risk of overfitting. Instead, we suggest focusing upon new diffusion encoding designs to disentangle anatomical features already at the acquisition as exemplified with multidimensional diffusion encoding (MDE) used for $\mu \mathrm{FA}$. This can also reduce modelling complexity.

An inspiration for future method development and application of high potential is to identify tissue types or pathologies with a substantial contrast in dMRI data and design diffusion encodings to separate those gross signal alterations into more qualitatively different microstructures. Exciting and promising examples along those lines is the prediction of chronic tissue damage in strokes from the kurtosis terms of the diffusion signal (Cheung et al., 2012; Wu et al., 2016), stratification of tumour cell morphology with microscopic anisotropy (Szczepankiewicz et al., 2015), and isolation of granular cell layers in hippocampus and cerebellum with time-dependent diffusion (Aggarwal et al., 2012; Lundell et al., 2015).

The large efforts in DDE and MDE methods exploring correlations of diffusion displacements in multiple dimensions is one example of how more independent information can be fed into our interpretations and models (Shemesh et al., 2016; Topgaard, 2017). Other examples of methods exploring correlations between multiple measurement dimensions include diffusion-weighted spectroscopy (DWS) to identify cell type specific metabolites or experiments exploring the correlation between diffusion and relaxation or magnetization transfer effects (Palombo et al., 2017; de Almeida Martins and Topgaard, 2018; Ronen et al., 2006; De Santis et al., 2016).

We all are in the same boat - data sharing initiatives. The potential of non-invasive microstructural imaging with dMRI and brain network mapping using tractography is unquestionably of high potential. The effective distribution of the new methods and simulation tools has for a long time been driven by an open access mentality in software development and easy-to-use software packages. Since the reliability and model fitting robustness of these methods goes hand in hand with the design of MRI and scan parameters, it stresses an urgent need for a similar open access mentality of sequences as well as of high quality MRI and validation material. Several larger data sharing initiatives of in vivo human MRI data have been launched e.g. the Human Connectome Project. $^{3}$ These studies uniquely include additional demographic profiling and behavioural test but miss the validation methods needed for microstructural imaging. Therefore, we emphasize the importance of closing this gap by also sharing the histological data as well as ex vivo MRI that only relatively few groups in the world can generate. A few validation data sharing initiatives have been established such as the tracer connectivity matrix ${ }^{4}$ by Markov et al. (2012), The CoCoMac database $^{5}$ (Bakker et al., 2011), The Allen Mouse Brain Connectivity Atlas $^{6}$ (Oh et al., 2014) and the White Matter Microscopy Database 7 under the open-science framework (https://osf.io/). The latter data sharing initiative is special for two reasons: (i) It both contains raw MRI data (mostly ex vivo) and raw histological data, and (ii) it is based on a data sharing initiative where research groups for free can upload their data. Several researchers have already done so. It pays off when sharing data leads to increased exposure of your research, increased number of citations, and, most importantly, the added value in the synergy from other researcher's ideas. We hope that those databases will grow with both more contributions and users.

In summary, to accelerate this highly needed development and to highlight the collaborative effort needed for validation, we encourage initiatives to make experimental protocols, multimodal data and analysis tools available to the community.

\section{Acknowledgement}

TD was supported by the Capital Region Research Foundation (grant number: A5657), HL was supported by the Danish Council for Independent Research (4093-00280A and 4093-00280B).

\section{References}

Aboiti, F., Scheibel, A.B., Fisher, R.S., Zaidel, E., 1992. Fiber composition of the human corpus callosum. Brain Res. 598, 143-153.

Aboitiz, F., Rodríguez, E., Olivares, R., Zaidel, E., 1996. Age-related changes in fibre composition of the human corpus callosum: sex differences. Neuroreport 7 , 1761-1764.

Aboitiz, F., Scheibel, A.B., Fisher, R.S., Zaidel, E., 1992. Individual differences in brain asymmetries and fiber composition in the human corpus callosum. Brain Res. 598, 154-161.

Aggarwal, M., Jones, M.V., Calabresi, P.A., Mori, S., Zhang, J., 2012. Probing mouse brain microstructure using oscillating gradient diffusion MRI. Magn. Reson. Med. 67, 98-109.

Alexander, D.C., 2008. A general framework for experiment design in diffusion MRI and its application in measuring direct tissue-microstructure features. Magn. Reson. Med. 60, 439-448.

Alexander, D.C., 2005. Multiple-fiber reconstruction algorithms for diffusion MRI. Ann. N. Y. Acad. Sci. 1064, 113-133.

Alexander, D.C., Dyrby, T.B., Nilsson, M., Zhang, H., 2017. Imaging brain microstructure with diffusion MRI: practicality and applications. NMR Biomed. https://doi.org/10. 1002/nbm.3841.

Alexander, D.C., Hubbard, P.L., Hall, M.G., Moore, E.A., Ptito, M., Parker, G.J.M., Dyrby, T.B., 2010. Orientationally invariant indices of axon diameter and density from diffusion MRI. Neuroimage 52, 1374-1389.

Aoki, H., 2017. Conformation and dynamics of single polymer chain studied by optical microscopy techniques beyond the diffraction limit. Microscopy 66, 223-233.

Assaf, Y., Alexander, D.C., Jones, D.K., Bizzi, A., Behrens, T.E.J., Clark, C.A., Cohen, Y., Dyrby, T.B., Huppi, P.S., Knoesche, T.R., Lebihan, D., Parker, G.J.M., Poupon, C., CONNECT consortium, Anaby, D., Anwander, A., Bar, L., Barazany, D., BlumenfeldKatzir, T., De-Santis, S., Duclap, D., Figini, M., Fischi, E., Guevara, P., Hubbard, P., Hofstetter, S., Jbabdi, S., Kunz, N., Lazeyras, F., Lebois, A., Liptrot, M.G., Lundell, H., Mangin, J.-F., Dominguez, D.M., Morozov, D., Schreiber, J., Seunarine, K., Nava, S., Poupon, C., Riffert, T., Sasson, E., Schmitt, B., Shemesh, N., Sotiropoulos, S.N.,

Tavor, I., Zhang, H.G., Zhou, F.-L., 2013. The CONNECT project: combining macroand micro-structure. Neuroimage 80, 273-282.

Assaf, Y., Blumenfeld-Katzir, T., Yovel, Y., Basser, P.J., 2008. AxCaliber: a method for measuring axon diameter distribution from diffusion MRI. Magn. Reson. Med. 59, 1347-1354.

\footnotetext{
${ }^{3}$ Human Connectome Project: https://www.humanconnectome.org/.

4 Tracer connectivity matrix: http://core-nets.org.

5 The CoCoMac 2.0 database: cocomac.g-node.org.

6 Allen Mouse Brain Connectivity Atlas: http://connectivity.brain-map.org/.

7 White Matter Microscopy Database: https://osf.io/yp4qg/
} 
Assaf, Y., Pasternak, O., 2008. Diffusion tensor imaging (DTI)-based white matter mapping in brain research: a review. J. Mol. Neurosci. 34, 51-61.

Bakker, R., Potjans, T.C., Wachtler, T., Diesmann, M., 2011. Macaque structural connectivity revisited: CoCoMac 2.0. BMC Neurosci. 12, P72.

Bakker, R., Wachtler, T., Diesmann, M., 2012. CoCoMac 2.0 and the future of tract-tracing databases. Front. Neuroinf. 6, 30.

Bartels, M., Krenkel, M., Cloetens, P., Möbius, W., Salditt, T., 2015. Myelinated mouse nerves studied by X-ray phase contrast zoom tomography. J. Struct. Biol. 192, $561-568$.

Basser, P.J., Mattiello, J., LeBihan, D., 1994. MR diffusion tensor spectroscopy and imaging. Biophys. J. 66, 259-267.

Basser, P.J., Pierpaoli, C., 1996. Microstructural and physiological features of tissues elucidated by quantitative-diffusion-tensor MRI. J. Magn. Reson. B 111, 209-219.

Beaulieu, C., 2002. The basis of anisotropic water diffusion in the nervous system - a technical review. NMR Biomed. 15, 435-455.

Behrens, T.E.J., Berg, H.J., Jbabdi, S., Rushworth, M.F.S., Woolrich, M.W., 2007 Probabilistic diffusion tractography with multiple fibre orientations: what can we gain? Neuroimage 34, 144-155.

Beier, K.T., Mundell, N.A., Pan, Y.A., Cepko, C.L., 2016 Jan 4. Anterograde or retrograde transsynaptic circuit tracing in vertebrates with vesicular stomatitis virus vectors. Curr. Protoc. Neurosci. 74 (1.26), 1-27. https://doi.org/10.1002/ 0471142301.ns0126s74.

Beltran, M.A., Paganin, D.M., Siu, K.K.W., Fouras, A., Hooper, S.B., Reser, D.H. Kitchen, M.J., 2011. Interface-specific x-ray phase retrieval tomography of complex biological organs. Phys. Med. Biol. 56, 7353-7369.

Benjamini, D., Komlosh, M.E., Holtzclaw, L.A., Nevo, U., Basser, P.J., 2016. White matter microstructure from nonparametric axon diameter distribution mapping. Neuroimage 135, 333-344.

Bentivoglio, M., Kuypers, H.G., 1982. Divergent axon collaterals from rat cerebellar nuclei to diencephalon, mesencephalon, medulla oblongata and cervical cord. A fluorescent double retrograde labeling study. Exp. Brain Res. 46, 339-356.

Bentivoglio, M., Kuypers, H.G.J.M., Catsman-Berrevoets, C.E., Dann, O., 1979. Fluorescent retrograde neuronal labeling in rat by means of substances binding specifically to adeninethymine rich DNA. Neurosci. Lett. 12, 235-240.

Brauckmann, S., 2004. The virtue of being too early: Paul A. Weiss and "axonal transport. Hist. Philos. Life Sci. 26, 333-353.

Budde, M.D., Annese, J., 2013. Quantification of anisotropy and fiber orientation in human brain histological sections. Front. Integr. Neurosci. 7, 3.

Budde, M.D., Frank, J.A., 2012. Examining brain microstructure using structure tensor analysis of histological sections. Neuroimage 63,1-10.

Budde, M.D., Kim, J.H., Liang, H.-F., Russell, J.H., Cross, A.H., Song, S.-K., 2008. Axona injury detected by in vivo diffusion tensor imaging correlates with neurological disability in a mouse model of multiple sclerosis. NMR Biomed. 21, 589-597.

Cahill, L.S., Laliberté, C.L., Ellegood, J., Spring, S., Gleave, J.A., Eede, M.C., van, Lerch, J.P., Henkelman, R.M., 2012. Preparation of fixed mouse brains for MRI Neuroimage 60, 933-939.

Calabrese, E., Badea, A., Cofer, G., Qi, Y., Allan Johnson, G., 2015. A diffusion MR tractography connectome of the mouse brain and comparison with neuronal tracer data. Cerebr. Cortex 25, 4628-4637.

Caminiti, R., Ghaziri, H., Galuske, R., Hof, P.R., Innocenti, G.M., 2009. Evolution amplified processing with temporally dispersed slow neuronal connectivity in primates. Proc. Natl. Acad. Sci. U. S. A 106, 19551-19556.

Campbell, J.S.W., Leppert, I.R., Narayanan, S., Boudreau, M., Duval, T., Cohen-Adad, J., Pike, G.B., Stikov, N., 2017. Promise and pitfalls of g-ratio estimation with MRI. Neuroimage. https://doi.org/10.1016/j.neuroimage.2017.08.038,

Cercignani, M., Embleton, K., Parker, G.J.M., Bozzali, M., 2012. Group-averaged anatomical connectivity mapping for improved human white matter pathway visualisation. NMR Biomed. 25, 1224-1233.

Chamberland, M., Bernier, M., Fortin, D., Whittingstall, K., Descoteaux, M., 2015. 3D interactive tractography-informed resting-state fMRI connectivity. Front. Neurosci. 9 275.

Chang, E.H., Argyelan, M., Aggarwal, M., Chandon, T.-S.S., Karlsgodt, K.H., Mori, S., Malhotra, A.K., 2017. The role of myelination in measures of white matter integrity: combination of diffusion tensor imaging and two-photon microscopy of CLARITY intact brains. Neuroimage 147, 253-261.

Cheung, J.S., Wang, E., Lo, E.H., Sun, P.Z., 2012. Stratification of heterogeneous diffusion MRI ischemic lesion with kurtosis imaging: evaluation of mean diffusion and kurtosis MRI mismatch in an animal model of transient focal ischemia. Stroke 43, 2252-2254.

Colello, R.J., Pott, U., Schwab, M.E., 1994. The role of oligodendrocytes and myelin on axon maturation in the developing rat retinofugal pathway. J. Neurosci. 14, 2594-2605.

Conturo, T.E., Lori, N.F. Cull, T.S., Akbudak, E., Snyder, A.Z., Shimony, J.S., McKinstry, R.C., Burton, H., Raichle, M.E., 1999. Tracking neuronal fiber pathways in the living human brain. Proc. Natl. Acad. Sci. U. S. A 96, 10422-10427.

Cullheim, S., Ulfhake, B., 1979. Relations between cell body size, axon diameter and axon conduction velocity of triceps surae alpha montoneurons during the postnatal development in the cat. J. Comp. Neurol. 188, 679-686.

D'Arceuil, H.E., Westmoreland, S., de Crespigny, A.J., 2007. An approach to high resolution diffusion tensor imaging in fixed primate brain. Neuroimage 35, 553-565.

Dauguet, J., Peled, S., Berezovskii, V., Delzescaux, T., Warfield, S.K., Born, R., Westin, C. F., 2007. Comparison of fiber tracts derived from in-vivo DTI tractography with 3D histological neural tract tracer reconstruction on a macaque brain. Neuroimage 37, $530-538$.

Davis, I., 2009. The "super-resolution" revolution. Biochem. Soc. Trans. 37, 1042-1044. de Almeida Martins, J.P., Topgaard, D., 2018. Multidimensional correlation of nuclear relaxation rates and diffusion tensors for model-free investigations of heterogeneous anisotropic porous materials. Sci. Rep. 8, 2488.

DeLuca, G.C., Ebers, G.C., Esiri, M.M., 2004. Axonal loss in multiple sclerosis: a pathological survey of the corticospinal and sensory tracts. Brain 127, 1009-1018.

De Santis, S., Barazany, D., Jones, D.K., Assaf, Y., 2016. Resolving relaxometry and diffusion properties within the same voxel in the presence of crossing fibres by combining inversion recovery and diffusion-weighted acquisitions. Magn. Reson. Med. 75, 372-380.

Donahue, C.J., Sotiropoulos, S.N., Jbabdi, S., Hernandez-Fernandez, M., Behrens, T.E., Dyrby, T.B., Coalson, T., Kennedy, H., Knoblauch, K., Van Essen, D.C., Glasser, M.F., 2016. Using diffusion tractography to predict cortical connection strength and distance: a quantitative comparison with tracers in the monkey. J. Neurosci. 36, 6758-6770.

Dučić, T., Quintes, S., Nave, K.-A., Susini, J., Rak, M., Tucoulou, R., Alevra, M., Guttmann, P., Salditt, T., 2011. Structure and composition of myelinated axons: a multimodal synchrotron spectro-microscopy study. J. Struct. Biol. 173, 202-212.

Dyrby, T.B., Baaré, W.F.C., Alexander, D.C., Jelsing, J., Garde, E., Søgaard, L.V., 2011. An ex vivo imaging pipeline for producing high-quality and high-resolution diffusionweighted imaging datasets. Hum. Brain Mapp. 32, 544-563.

Dyrby, T.B., Burke, M., Alexander, D.C., Ptito, M., 2014a. Undulating and crossing axons in the corpus callosum may explain overstimation of axon diameters with ActiveAx. In: International Society for Magnetic Resonance in Medicine. Abstract number: 2619.

Dyrby, T.B., Lundell, H., Burke, M.W., Reislev, N.L., Paulson, O.B., Ptito, M., Siebner, H.R., 2014b. Interpolation of diffusion weighted imaging datasets. Neuroimage 103, 202-213.

Dyrby, T.B., Sogaard, L.V., Hall, M.G., Ptito, M., Alexander, D.C., 2013. Contrast and stability of the axon diameter index from microstructure imaging with diffusion MRI. Magn. Reson. Med. 70, 711-721.

Dyrby, T.B., Søgaard, L.V., Parker, G.J., Alexander, D.C., Lind, N.M., Baaré, W.F.C., HaySchmidt, A., Eriksen, N., Pakkenberg, B., Paulson, O.B., Jelsing, J., 2007. Validation of in vitro probabilistic tractography. Neuroimage 37, 1267-1277.

Edgar, J.M., Griffiths, I.R., 2009. Chapter 5-white matter structure: a Microscopist's view. In: Johansen-Berg, H., Behrens, T.E.J. (Eds.), Diffusion MRI. Academic Press, San Diego, pp. 74-103.

Firmin, L., Field, P., Maier, M.A., Kraskov, A., Kirkwood, P.A., Nakajima, K., Lemon, R.N. Glickstein, M., 2014. Axon diameters and conduction velocities in the macaque pyramidal tract. J. Neurophysiol. 112, 1229-1240.

Fitzgerald, R., 2000. Phase-sensitive x-ray imaging. Phys. Today 53, 23-26.

Fling, B.W., Benson, B.L., Seidler, R.D., 2013. Transcallosal sensorimotor fiber tract structure-function relationships. Hum. Brain Mapp. 34, 384-395.

Flint, J., Hansen, B., Vestergaard-Poulsen, P., Blackband, S.J., 2009. Diffusion weighted magnetic resonance imaging of neuronal activity in the hippocampal slice model. Neuroimage 46, 411-418.

Flint, J.J., Hansen, B., Fey, M., Schmidig, D., King, M.A., Vestergaard-Poulsen, P., Blackband, S.J., 2010. Cellular-level diffusion tensor microscopy and fiber tracking in mammalian nervous tissue with direct histological correlation. Neuroimage 52, $556-561$.

Ford, M.C., Alexandrova, O., Cossell, L., Stange-Marten, A., Sinclair, J., KoppScheinpflug, C., Pecka, M., Attwell, D., Grothe, B., 2015. Tuning of Ranvier node and internode properties in myelinated axons to adjust action potential timing. Nat. Commun. 6, 8073.

Fox, C.H., Johnson, F.B., Whiting, J., Roller, P.P., 1985. Formaldehyde fixation. J. Histochem. Cytochem. 33, 845-853.

Fritzsch, B., Wilm, C., 1990. Dextran amines in neuronal tracing. Trends Neurosci. 13, 14.

Galea, M.P., Darian-Smith, I., 1995. Postnatal maturation of the direct corticospinal projections in the macaque monkey. Cerebr. Cortex 5, 518-540.

Goldberg, J.L., 2003. How does an axon grow? Genes Dev. 17, 941-958.

Groppa, S., Muthuraman, M., Otto, B., Deuschl, G., Siebner, H.R., Raethjen, J., 2013 Subcortical substrates of TMS induced modulation of the cortico-cortical connectivity. Brain Stimul 6, 138-146.

Grussu, F., Schneider, T., Tur, C., Yates, R.L., Tachrount, M., Ianuş, A., Yiannakas, M.C., Newcombe, J., Zhang, H., Alexander, D.C., DeLuca, G.C., Gandini WheelerKingshott, C.A.M., 2017. Neurite dispersion: a new marker of multiple sclerosis spinal cord pathology? Ann Clin Transl Neurol 4, 663-679.

Hansen, B., Flint, J.J., Heon-Lee, C., Fey, M., Vincent, F., King, M.A., VestergaardPoulsen, P., Blackband, S.J., 2011. Diffusion tensor microscopy in human nervous tissue with quantitative correlation based on direct histological comparison. Neuroimage 57, 1458-1465.

Hansen, J.S., Bruus, H., Todd, B.D., Daivis, P.J., 2010. Rotational and spin viscosities of water: application to nanofluidics. J. Chem. Phys. 133, 144906.

Hau, J., Sarubbo, S., Houde, J.C., Corsini, F., Girard, G., Deledalle, C., Crivello, F., Zago, L., Mellet, E., Jobard, G., Joliot, M., Mazoyer, B., Tzourio-Mazoyer, N., Descoteaux, M., Petit, L., 2017. Revisiting the human uncinate fasciculus, its subcomponents and asymmetries with stem-based tractography and microdissection validation. Brain Struct. Funct. 222, 1645-1662.

Heimer, L., Robards, M.J. (Eds.), 1981. Neuroanatomical Tract-tracing Methods. Plenum Press, New York.

Honig, M.G., Hume, R.I., 1989. Dil and diO: versatile fluorescent dyes for neuronal labelling and pathway tracing. Trends Neurosci. 12, 333-335, 340-1.

Horowitz, A., Barazany, D., Tavor, I., Bernstein, M., Yovel, G., Assaf, Y., 2014. In vivo correlation between axon diameter and conduction velocity in the human brain. Brain Struct. Funct. 220, 1777-1788. 
Houzel, J.C., Milleret, C., Innocenti, G., 1994. Morphology of callosal axons interconnecting areas 17 and 18 of the cat. Eur. J. Neurosci. 6, 898-917.

Hukkanen, V., Röyttä, M., 1987. Autolytic changes of human white matter: an electron microscopic and electrophoretic study. Exp. Mol. Pathol. 46, 31-39.

Hursh, J.B., 1939. Conduction velocity and diameter of nerve fibers. Am Physiological Soc $127,131-139$.

Innocenti, G.M., 2017. Network causality, axonal computations, and Poffenberger. Exp. Brain Res. 235, 2349-2357.

Innocenti, G.M., 2002. Architecture and callosal connections of visual areas 17, 18, 19 and 21 in the ferret (Mustela putorius). Cerebr. Cortex 12, 411-422.

Innocenti, G.M., 1981. Growth and reshaping of axons in the establishment of visual callosal connections. Science 212, 824-827.

Innocenti, G.M., Caminiti, R., 2017. Axon diameter relates to synaptic bouton size: structural properties define computationally different types of cortical connections in primates. Brain Struct. Funct. 222, 1169-1177.

Innocenti, G.M., Caminiti, R., Aboitiz, F., 2015. Comments on the paper by Horowitz et al. (2014). Brain Struct. Funct. 220, 1789-1790.

Innocenti, G.M., Carlén, M., Dyrby, T.B., 2016. The diameters of cortical axons and their relevance to neural computing. In: Axons and Brain Architecture, pp. 317-335.

Innocenti, G.M., Dyrby, T.B., Andersen, K.W., Rouiller, E.M., Caminiti, R., 2017. The crossed projection to the striatum in two species of monkey and in humans: behavioral and evolutionary significance. Cerebr. Cortex 27, 3217-3230.

Innocenti, G.M., Fiore, L., Caminiti, R., 1977. Exuberant projection into the corpus callosum from the visual cortex of newborn cats. Neurosci. Lett. 4, 237-242.

Innocenti, G.M., Price, D.J., 2005. Exuberance in the development of cortical networks. Nat. Rev. Neurosci. 6, 955-965.

Innocenti, G.M., Vercelli, A., Caminiti, R., 2014. The diameter of cortical axons depends both on the area of origin and target. Cerebr. Cortex 24, 2178-2188.

Inoue, T., Majid, T., Pautler, R.G., 2011. Manganese enhanced MRI (MEMRI): neurophysiological applications. Rev. Neurosci. 22, 675-694.

Jbabdi, S., Johansen-Berg, H., 2011. Tractography: where do we go from here? Brain Connect. 1, 169-183.

Jbabdi, S., Lehman, J.F., Haber, S.N., Behrens, T.E., 2013. Human and monkey ventral prefrontal fibers use the same organizational principles to reach their targets: tracing versus tractography. J. Neurosci. 33, 3190-3201.

Jelescu, I.O., Veraart, J., Fieremans, E., Novikov, D.S., 2016. Degeneracy in model parameter estimation for multi-compartmental diffusion in neuronal tissue. NMR Biomed. 29, 33-47.

Jespersen, S.N., Bjarkam, C.R., Nyengaard, J.R., Chakravarty, M.M., Hansen, B., Vosegaard, T., Østergaard, L., Yablonskiy, D., Nielsen, N.C., Vestergaard-Poulsen, P., 2010. Neurite density from magnetic resonance diffusion measurements at ultrahigh field: comparison with light microscopy and electron microscopy. Neuroimage 49, 205-216.

Jespersen, S.N., Leigland, L.A., Cornea, A., Kroenke, C.D., 2012. Determination of axona and dendritic orientation distributions within the developing cerebral cortex by diffusion tensor imaging. IEEE Trans. Med. Imag. 31, 16-32.

Jespersen, S.N., Lundell, H., Sønderby, C.K., Dyrby, T.B., 2013. Orientationally invarian metrics of apparent compartment eccentricity from double pulsed field gradient diffusion experiments. NMR Biomed. 26, 1647-1662.

Jeurissen, B., Descoteaux, M., Mori, S., Leemans, A., 2017. Diffusion MRI fiber tractography of the brain. NMR Biomed. https://doi.org/10.1002/nbm.3785.

Jones, D.K., Cercignani, M., 2010. Twenty-five pitfalls in the analysis of diffusion MRI data. NMR Biomed. 23, 803-820.

Kaden, E., Kelm, N.D., Carson, R.P., Does, M.D., Alexander, D.C., 2016. Multicompartment microscopic diffusion imaging. Neuroimage 139, 346-359.

Kakkar, L.S., Bennett, O.F., Siow, B., Richardson, S., Ianuş, A., Quick, T., Atkinson, D., Phillips, J.B., Drobnjak, I., 2017. Low frequency oscillating gradient spin-echo sequences improve sensitivity to axon diameter: an experimental study in viable nerve tissue. Neuroimage. https://doi.org/10.1016/j.neuroimage.2017.07.060.

Kamagata, K., Kerever, A., Yokosawa, S., Otake, Y., Ochi, H., Hori, M., Kamiya, K. Tsuruta, K., Tagawa, K., Okazawa, H., Aoki, S., Arikawa-Hirasawa, E., 2016. Quantitative histological validation of diffusion tensor MRI with two-photon microscopy of cleared mouse brain. Magn. Reson. Med. Sci. 15, 416-421.

Kasthuri, N., Hayworth, K.J., Berger, D.R., Schalek, R.L., Conchello, J.A., KnowlesBarley, S., Lee, D., Vázquez-Reina, A., Kaynig, V., Jones, T.R., Roberts, M., Morgan, J.L., Tapia, J.C., Seung, H.S., Roncal, W.G., Vogelstein, J.T., Burns, R., Sussman, D.L., Priebe, C.E., Pfister, H., Lichtman, J.W., 2015. Saturated reconstruction of a volume of neocortex. Cell 162, 648-661.

Katz, L.C., Burkhalter, A., Dreyer, W.J., 1984. Fluorescent latex microspheres as retrograde neuronal marker for in vivo and in vitro studies of visual cortex. Nature $310,498-500$.

Khan, A.R., Chuhutin, A., Wiborg, O., Kroenke, C.D., Nyengaard, J.R., Hansen, B., Jespersen, S.N., 2016. Biophysical modeling of high field diffusion MRI demonstrate micro-structural aberration in chronic mild stress rat brain. Neuroimage 142, $421-430$.

Khan, A.R., Cornea, A., Leigland, L.A., Kohama, S.G., Jespersen, S.N., Kroenke, C.D., 2015. 3D structure tensor analysis of light microscopy data for validating diffusion MRI. Neuroimage 111, 192-203.

King, M.A., Louis, P.M., Hunter, B.E., Walker, D.W., 1989. Biocytin: a versatile anterograde neuroanatomical tract-tracing alternative. Brain Res. 497, 361-367.

Knösche, T.R., Anwander, A., Liptrot, M., Dyrby, T.B., 2015. Validation of tractography: comparison with manganese tracing. Hum. Brain Mapp. 36, 4116-4134.

Kristensson, K., Olsson, Y., 1971. Retrograde axonal transport of protein. Brain Res. 29, 363-365.

Lamantia, A.S., Rakic, P., 1990a. Cytological and quantitative characteristics of four cerebral commissures in the rhesus monkey. J. Comp. Neurol. 291, 520-537.
LaMantia, A.S., Rakic, P., 1990b. Axon overproduction and elimination in the corpus callosum of the developing rhesus monkey. J. Neurosci. 10, 2156-2175.

Lasič, S., Nilsson, M., Lätt, J., Ståhlberg, F., Topgaard, D., 2011. Apparent exchange rate mapping with diffusion MRI. Magn. Reson. Med. 66, 356-365.

Lasič, S., Szczepankiewicz, F., Eriksson, S., Nilsson, M., Topgaard, D., 2014 Microanisotropy imaging: quantification of microscopic diffusion anisotropy and orientational order parameter by diffusion MRI with magic-angle spinning of the qvector. Frontiers in Physics 2. https://doi.org/10.3389/fphy.2014.00011.

Le Bihan, D., Delannoy, J., Levin, R.L., 1989. Temperature mapping with MR imaging of molecular diffusion: application to hyperthermia. Radiology 171, 853-857.

Leergaard, T.B., White, N.S., de Crespigny, A., Bolstad, I., D'Arceuil, H., Bjaalie, J.G., Dale, A.M., 2010. Quantitative histological validation of diffusion MRI fiber orientation distributions in the rat brain. PLoS One 5, e8595.

Leprince, Y., Schmitt, B., Chaillou, E., Destrieux, C., Barantin, L., Vignaud, A., Rivière, D., Poupon, C., 2015. Optimization of sample preparation for MRI of formaldehyde-fixed brains. Presented at the International Society for Magnetic Resonance in Medicine 2283.

LeVay, S., 1988. The patchy intrinsic projections of visual cortex. Prog. Brain Res. 75, 147-161.

LeVay, S., Wiesel, T.N., Hubel, D.H., 1980. The development of ocular dominance columns in normal and visually deprived monkeys. J. Comp. Neurol. 191, 1-51.

Lind, N.M., Moustgaard, A., Jelsing, J., Vajta, G., Cumming, P., Hansen, A.K., 2007. The use of pigs in neuroscience: modeling brain disorders. Neurosci. Biobehav. Rev. 31, 728-751.

Liptrot, M.G., Sidaros, K., Dyrby, T.B., 2014. Addressing the path-length-dependency confound in white matter tract segmentation. PLoS One 9, e96247.

Lundell, H., Nielsen, J.B., Ptito, M., Dyrby, T.B., 2011. Distribution of collateral fibers in the monkey cervical spinal cord detected with diffusion-weighted magnetic resonance imaging. Neuroimage 56, 923-929.

Lundell, H., Nilsson, M., Dyrby, T.B., Parker, P.G., Cristinacce, P., Zhou, F., Topgaard, D., Lasic, S., 2017. Microscopic Anisotropy with Spectrally Modulated Q-space Trajectory Encoding. International Society for Magnetic Resonance in Medicine.

Lundell, H., Sønderby, C.K., Dyrby, T.B., 2015. Diffusion weighted imaging with circularly polarized oscillating gradients. Magn. Reson. Med. 73, 1171-1176.

Luo, L., O'Leary, D.D.M., 2005. Axon retraction and degeneration in development and disease. Annu. Rev. Neurosci. 28, 127-156.

Lyksborg, M., Siebner, H.R., Sørensen, P.S., Blinkenberg, M., Parker, G.J.M. Dogonowski, A.-M., Garde, E., Larsen, R., Dyrby, T.B., 2014. Secondary progressive and relapsing remitting multiple sclerosis leads to motor-related decreased anatomical connectivity. PLoS One 9, e95540.

Maier-Hein, K.H., Neher, P.F., Houde, J.-C., Côté, M.-A., Garyfallidis, E., Zhong, J., Chamberland, M., Yeh, F.-C., Lin, Y.-C., Ji, Q., Reddick, W.E., Glass, J.O., Chen, D.Q., Feng, Y., Gao, C., Wu, Y., Ma, J., Renjie, H., Li, Q., Westin, C.-F., DeslauriersGauthier, S., González, J.O.O., Paquette, M., St-Jean, S., Girard, G., Rheault, F. Sidhu, J., Tax, C.M.W., Guo, F., Mesri, H.Y., Dávid, S., Froeling, M., Heemskerk, A.M., Leemans, A., Boré, A., Pinsard, B., Bedetti, C., Desrosiers, M., Brambati, S., Doyon, J., Sarica, A., Vasta, R., Cerasa, A., Quattrone, A., Yeatman, J., Khan, A.R., Hodges, W., Alexander, S., Romascano, D., Barakovic, M., Auría, A., Esteban, O., Lemkaddem, A., Thiran, J.-P., Cetingul, H.E., Odry, B.L., Mailhe, B., Nadar, M.S., Pizzagalli, F., Prasad, G., Villalon-Reina, J.E., Galvis, J., Thompson, P.M., Requejo, F.D.S., Laguna, P.L., Lacerda, L.M., Barrett, R., Dell'Acqua, F., Catani, M., Petit, L., Caruyer, E., Daducci, A., Dyrby, T.B., Holland-Letz, T., Hilgetag, C.C., Stieltjes, B., Descoteaux, M., 2017. The challenge of mapping the human connectome based on diffusion tractography. Nat. Commun. 8, 1349.

Markov, N.T., Ercsey-Ravasz, M.M., Ribeiro Gomes, A.R., Lamy, C., Magrou, L., Vezoli, J., Misery, P., Falchier, A., Quilodran, R., Gariel, M.A., Sallet, J., Gamanut, R., Huissoud, C., Clavagnier, S., Giroud, P., Sappey-Marinier, D., Barone, P., Dehay, C., Toroczkai, Z., Knoblauch, K., Van Essen, D.C., Kennedy, H., 2012. A weighted and directed interareal connectivity matrix for macaque cerebral cortex. Cerebr. Cortex 24, 17-36.

Martersteck, E.M., Hirokawa, K.E., Evarts, M., Bernard, A., Duan, X., Li, Y., Ng, L. Oh, S.W., Ouellette, B., Royall, J.J., Stoecklin, M., Wang, Q., Zeng, H., Sanes, J.R., Harris, J.A., 2017. Diverse central projection patterns of retinal ganglion cells. Cell Rep. 18 (8), 2058-2072. https://doi.org/10.1016/j.celrep.2017.01.075.

Martin, J.H., Choy, M., Pullman, S., Meng, Z., 2004. Corticospinal system development depends on motor experience. J. Neurosci. 24, 2122-2132.

Molnár, Z., Adams, R., Blakemore, C., 1998. Mechanisms underlying the early establishment of thalamocortical connections in the rat. J. Neurosci. 18, 5723-5745.

Mori, S., Crain, B.J., Chacko, V.P., Van Zijl, P., 1999. Three-dimensional tracking of axonal projections in the brain by magnetic resonance imaging. Ann. Neurol. 45 265-269.

Moseley, M.E., Kucharczyk, J, Mintorovitch, J., Cohen, Y., Kurhanewicz, J., Derugin, N., Asgari, H., Norman, D., 1990. Diffusion-weighted MR imaging of acute stroke: correlation with T2-weighted and magnetic susceptibility-enhanced MR imaging in cats. AJNR Am. J. Neuroradiol 11, 423-429.

Nilsson, M., Lasič, S., Drobnjak, I., Topgaard, D., Westin, C.-F., 2017. Resolution limit of cylinder diameter estimation by diffusion MRI: the impact of gradient waveform and orientation dispersion. NMR Biomed. 30. https://doi.org/10.1002/nbm.3711.

Nilsson, M., Lätt, J., Ståhlberg, F., Westen, D., Hagslätt, H., 2011. The importance of axonal undulation in diffusion MR measurements: a Monte Carlo simulation study. NMR Biomed. 25, 795-805.

Nilsson, M., Lätt, J., van Westen, D., Brockstedt, S., Lasič, S., Ståhlberg, F., Topgaard, D., 2013. Noninvasive mapping of water diffusional exchange in the human brain using filter-exchange imaging. Magn. Reson. Med. 69, 1573-1581.

Norris, C.R., Kalil, K., 1991. Guidance of callosal axons by radial glia in the developing cerebral cortex. J. Neurosci. 11, 3481-3492. 
Novikov, D.S., Kiselev, V.G., 2010. Effective medium theory of a diffusion-weighted signal. NMR Biomed. 23, 682-697.

Novikov, D.S., Kiselev, V.G., Jespersen, S.N., 2018. On modeling. Magn. Reson. Med. 79, 3172-3193.

Oh, S.W., Harris, J.A., Ng, L., Winslow, B., Cain, N., Mihalas, S., Wang, Q., Lau, C., Kuan, L., Henry, A.M., Mortrud, M.T., Ouellette, B., Nguyen, T.N., Sorensen, S.A., Slaughterbeck, C.R., Wakeman, W., Li, Y., Feng, D., Ho, A., Nicholas, E., Hirokawa, K.E., Bohn, P., Joines, K.M., Peng, H., Hawrylycz, M.J., Phillips, J.W., Hohmann, J.G., Wohnoutka, P., Gerfen, C.R., Koch, C., Bernard, A., Dang, C., Jones, A.R., Zeng, H., 2014. A mesoscale connectome of the mouse brain. Nature 508, 207-214.

Palombo, M., Ligneul, C., Najac, C., Le Douce, J., Flament, J., Escartin, C., Hantraye, P., Brouillet, E., Bonvento, G., Valette, J., 2016. New paradigm to assess brain cell morphology by diffusion-weighted MR spectroscopy in vivo. Proc. Natl. Acad. Sci. U. S. A $113,6671-6676$.

Palombo, M., Shemesh, N., Ronen, I., Valette, J., 2017. Insights into brain microstructure from in vivo DW-MRS. Neuroimage. https://doi.org/10.1016/j.neuroimage.2017.11. 028 .

Panagiotaki, E., Schneider, T., Siow, B., Hall, M.G., Lythgoe, M.F., Alexander, D.C., 2012 Compartment models of the diffusion MR signal in brain white matter: a taxonomy and comparison. Neuroimage 59, 2241-2254.

Pautler, R.G., 2004. In vivo, trans-synaptic tract-tracing utilizing manganese-enhanced magnetic resonance imaging (MEMRI). NMR Biomed. 17, 595-601.

Perge, J.A., Niven, J.E., Mugnaini, E., Balasubramanian, V., Sterling, P., 2012. Why do axons differ in caliber? J. Neurosci. 32, 626-638.

Ragan, T., Kadiri, L.R., Venkataraju, K.U., Bahlmann, K., Sutin, J., Taranda, J., ArgandaCarreras, I., Kim, Y., Seung, H.S., Osten, P., 2012. Serial two-photon tomography for automated ex vivo mouse brain imaging. Nat. Methods 9, 255-258.

Reveley, C., Seth, A.K., Pierpaoli, C., Silva, A.C., Yu, D., Saunders, R.C., Leopold, D.A., Ye, F.Q., 2015. Superficial white matter fiber systems impede detection of long-range cortical connections in diffusion MR tractography. Proc. Natl. Acad. Sci. Unit. States Am. 112, E2820-E2828.

Richardson, D.S., Lichtman, J.W., 2015. Clarifying tissue clearing. Cell 162, 246-257.

Riise, J., Pakkenberg, B., 2011. Stereological estimation of the total number of myelinated callosal fibers in human subjects. J. Anat. 218, 277-284.

Rilling, J.K., Glasser, M.F., Jbabdi, S., Andersson, J., Preuss, T.M., 2011. Continuity, divergence, and the evolution of brain language pathways. Front. Evol. Neurosci. 3 , 11.

Ronen, I., Ercan, E., Webb, A., 2013. Axonal and glial microstructural information obtained with diffusion-weighted magnetic resonance spectroscopy at 7T. Front. Integr. Neurosci. 7, 13.

Ronen, I., Moeller, S., Ugurbil, K., Kim, D.-S., 2006. Investigation of multicomponent diffusion in cat brain using a combined MTC-DWI approach. Magn. Reson. Imaging $24,425-431$.

Rushton, W.A.H., 1951. A theory of the effects of fibre size in medullated nerve. J. Physiol. (London) 115, 101-122.

Saleem, K.S., Pauls, J.M., Augath, M., Trinath, T., Prause, B.A., Hashikawa, T. Logothetis, N.K., 2002. Magnetic resonance imaging of neuronal connections in the macaque monkey. Neuron 34, 685-700.

Saliani, A., Perraud, B., Duval, T., Stikov, N., Rossignol, S., Cohen-Adad, J., 2017. Axon and myelin morphology in animal and human spinal cord. Front Neuroanat. 11, 129.

Salo, R.A., Belevich, I., Manninen, E., Jokitalo, E., Gröhn, O., Sierra, A., 2018. Quantification of anisotropy and orientation in 3D electron microscopy and diffusion tensor imaging in injured rat brain. Neuroimage 172, 404-414.

Schain, A.J., Hill, R.A., Grutzendler, J., 2014. Label-free in vivo imaging of myelinated axons in health and disease with spectral confocal reflectance microscopy. Nat. Med. 20, 443-449.

Schilling, K., Gao, Y., Janve, V., Stepniewska, I., Landman, B.A., Anderson, A.W., 2018. Confirmation of a gyral bias in diffusion MRI fiber tractography. Hum. Brain Mapp 39, 1449-1466.

Schilling, K., Gao, Y., Janve, V., Stepniewska, I., Landman, B.A., Anderson, A.W., 2017. Can increased spatial resolution solve the crossing fiber problem for diffusion MRI? NMR Biomed. 30. https://doi.org/10.1002/nbm.3787.

Schmahmann, J.D., Pandya, D.N., 2006. Fiber Pathways of the Brain. Oxford University press.

Schmahmann, J.D., Pandya, D.N., Wang, R., Dai, G., D'Arceuil, H.E., de Crespigny, A.J., Wedeen, V.J., 2007. Association fibre pathways of the brain: parallel observations from diffusion spectrum imaging and autoradiography. Brain 130, 630-653.

Schwartz, E.D., Duda, J., Shumsky, J.S., Cooper, E.T., Gee, J., 2005. Spinal cord diffusion tensor imaging and fiber tracking can identify white matter tract disruption and glia scar orientation following lateral funiculotomy. J. Neurotrauma 22, 1388-1398.

Sepehrband, F., Alexander, D.C., Kurniawan, N.D., Reutens, D.C., Yang, Z., 2016. Towards higher sensitivity and stability of axon diameter estimation with diffusion-weighted MRI. NMR Biomed. 29, 293-308.

Shemesh, N., Jespersen, S.N., Alexander, D.C., Cohen, Y., Drobnjak, I., Dyrby, T.B., Finsterbusch, J., Koch, M.A., Kuder, T., Laun, F., Lawrenz, M., Lundell, H., Mitra, P.P., Nilsson, M., Özarslan, E., Topgaard, D., Westin, C.-F., 2016. Conventions and nomenclature for double diffusion encoding NMR and MRI. Magn. Reson. Med. 75, $82-87$.
Shepherd, T.M., Thelwall, P.E., Stanisz, G.J., Blackband, S.J., 2009. Aldehyde fixative solutions alter the water relaxation and diffusion properties of nervous tissue. Magn. Reson. Med. 62, 26-34.

Silvestri, L., Costantini, I., Sacconi, L., Pavone, F.S., 2016. Clearing of fixed tissue: a review from a microscopist's perspective. J. Biomed. Opt. 21, 081205.

Sorby-Adams, A.J., Vink, R., Turner, R.J., 2018. Large animal models of stroke and traumatic brain injury as translational tools. Am. J. Physiol. Regul. Integr. Comp. Physiol. https://doi.org/10.1152/ajpregu.00163.2017.

Stanisz, G.J., Szafer, A., Wright, G.A., Henkelman, R.M., 1997. An analytical model of restricted diffusion in bovine optic nerve. Magn. Reson. Med. 37, 103-111.

Stephan, K.E., Kamper, L., Bozkurt, A., G A P, Young, M.P., Kotter, R., 2001. Advanced database methodology for the collation of connectivity data on the macaque brain (CoCoMac). Philos. Trans. R. Soc. Lond. B Biol. Sci. 356, 1159-1186.

St-Onge, E., Girard, G., Whittingstall, K., Descoteaux, M., 2015. Surface tracking from the cortical mesh complements diffusion MRI fiber tracking near the cortex. In: Presented at the International Society of Magnetic Resonance in Medicine (ISMRM), p. 5810.

Sun, S.-W., Neil, J.J., Liang, H.-F., He, Y.Y., Schmidt, R.E., Hsu, C.Y., Song, S.-K., 2005 Formalin fixation alters water diffusion coefficient magnitude but not anisotropy in infarcted brain. Magn. Reson. Med. 53, 1447-1451.

Sykova, E., Nicholson, C., 2008. Diffusion in brain extracellular space. Physiol. Rev. 88, 1277-1340.

Szczepankiewicz, F., Lasič, S., van Westen, D., Sundgren, P.C., Englund, E., Westin, C.-F., Ståhlberg, F., Lätt, J., Topgaard, D., Nilsson, M., 2015. Quantification of microscopic diffusion anisotropy disentangles effects of orientation dispersion from microstructure: applications in healthy volunteers and in brain tumors. Neuroimage $104,241-252$

Teh, I., McClymont, D., Zdora, M.-C., Whittington, H.J., Davidoiu, V., Lee, J., Lygate, C.A., Rau, C., Zanette, I., Schneider, J.E., 2017. Validation of diffusion tensor MRI measurements of cardiac microstructure with structure tensor synchrotron radiation imaging. J. Cardiovasc. Magn. Reson. 19, 31.

Tettoni, L., Gheorghita-Baechler, F., Bressoud, R., Welker, E., Innocenti, G.M., 1998. Constant and variable aspects of axonal phenotype in cerebral cortex. Cerebr. Cortex $8,543-552$.

Tettoni, L., Lehmann, P., Houzel, J.C., Innocenti, G.M., 1996. Maxsim, software for the analysis of multiple axonal arbors and their simulated activation. J. Neurosci. Meth. 67, 1-9.

Thomas, C., Ye, F.Q., Irfanoglu, M.O., Modi, P., Saleem, K.S., Leopold, D.A., Pierpaoli, C., 2014. Anatomical accuracy of brain connections derived from diffusion MRI tractography is inherently limited. Proc. Natl. Acad. Sci. U. S. A 111, 16574-16579.

Tomasi, S., Caminiti, R., Innocenti, G.M., 2012. Areal differences in diameter and length of corticofugal projections. Cerebr. Cortex 22, 1463-1472.

Topgaard, D., 2017. Multidimensional diffusion MRI. J. Magn. Reson. 275, 98-113.

Tournier, J.-D., Calamante, F., Connelly, A., 2012. MRtrix: diffusion tractography in crossing fiber regions. Int. J. Imag. Syst. Technol. 22, 53-66.

Tournier, J.-D., Mori, S., Leemans, A., 2011. Diffusion tensor imaging and beyond. Magn. Reson. Med. 65, 1532-1556.

Tovi, M., Ericsson, A., 1992. Measurements of T1 and T2 over time in formalin-fixed human whole-brain specimens. Acta Radiol. 33, 400-404.

van den Heuvel, M.P., de Reus, M.A., Feldman Barrett, L., Scholtens, L.H. Coopmans, F.M.T., Schmidt, R., Preuss, T.M., Rilling, J.K., Li, L., 2015. Comparison of diffusion tractography and tract-tracing measures of connectivity strength in rhesus macaque connectome. Hum. Brain Mapp. 36, 3064-3075.

Vercelli, A., Repici, M., Garbossa, D., Grimaldi, A., 2000. Recent techniques for tracing pathways in the central nervous system of developing and adult mammals. Brain Res. Bull. 51, 11-28.

Weiskopf, N., Suckling, J., Williams, G., Correia, M.M., Inkster, B., Tait, R., Ooi, C., Bullmore, E.T., Lutti, A., 2013. Quantitative multi-parameter mapping of R1, PD*, MT, and R2* at 3T: a multi-center validation. Front. Neurosci. 7. https://doi.org/10. 3389/fnins.2013.00095.

Westin, C.-F., Maier, S.E., Mamata, H., Nabavi, A., Jolesz, F.A., Kikinis, R., 2002. Processing and visualization for diffusion tensor MRI. Med. Image Anal. 6, 93-108.

Wu, Y., Kim, J., Chan, S.-T., Zhou, I.Y., Guo, Y., Igarashi, T., Zheng, H., Guo, G., Sun, P.Z., 2016. Comparison of image sensitivity between conventional tensor-based and fast diffusion kurtosis imaging protocols in a rodent model of acute ischemic stroke. NMR Biomed. 29, 625-630.

Yang, G., Tian, Q., Leuze, C., Wintermark, M., McNab, J.A., 2018. Double diffusion encoding MRI for the clinic. Magn. Reson. Med. 80, 507-520.

y Cajal, S.R., 1995. Histology of the nervous system of man and vertebrates.

Zaborszky, L., Wouterlood, F.G., Lanciego, J.L. (Eds.), 2006. Neuroanatomical Tracttracing: Molecules, Neurons, and Systems. Springer.

Zhang, H., Dyrby, T.B., Alexander, D.C., 2011a. Axon diameter mapping in crossing fibers with diffusion MRI. In: Lecture Notes in Computer Science, pp. 82-89.

Zhang, H., Hubbard, P.L., Parker, G.J.M., Alexander, D.C., 2011b. Axon diameter mapping in the presence of orientation dispersion with diffusion MRI. Neuroimage $56,1301-1315$.

Zhang, H., Schneider, T., Wheeler-Kingshott, C.A., Alexander, D.C., 2012. NODDI: practical in vivo neurite orientation dispersion and density imaging of the human brain. Neuroimage 61, 1000-1016. 